\title{
Segregation of Flowing Blood: Mathematical Description
}

\author{
A. Tokarev $^{1 *}$, G. Panasenko ${ }^{2}$ and F. Ataullakhanov ${ }^{1}$ \\ ${ }^{1}$ National Research Center for Hematology, Russian Academy of Medical Sciences \\ Novii Zykovskii proezd, 4a, Moscow, Russia, 125167 \\ ${ }^{2}$ University Jean Monnet, 23 rue Dr. Paul Michelon, 42023 Saint-Etienne, France
}

\begin{abstract}
Blood rheology is completely determined by its major corpuscles which are erythrocytes, or red blood cells (RBCs). That is why understanding and correct mathematical description of RBCs behavior in blood is a critical step in modelling the blood dynamics. Various phenomena provided by RBCs such as aggregation, deformation, shear-induced diffusion and non-uniform radial distribution affect the passage of blood through the vessels. Hence, they have to be taken into account while modelling the blood dynamics. Other important blood corpuscles are platelets, which are crucial for blood clotting. RBCs strongly affect the platelet transport in blood expelling them to the vessel walls and increasing their dispersion, which has to be considered in models of clotting. In this article we give a brief review of basic modern approaches in mathematical description of these phenomena, discuss their applicability to real flow conditions and propose further pathways for developing the theory of blood flow.
\end{abstract}

Key words: blood, erythrocytes, platelets, suspension dynamics

AMS subject classification: 9202, 92C10, 92C35, 76Z05

\section{Introduction}

Blood is a biological fluid circulating in the vascular system. Its main function is to transport oxygen throughout the body. Blood flows from heart through aorta, arteries and arterioles to capillaries and back to the heart through venules and veins. Interdisciplinary investigations of the processes

*Corresponding author. E-mail: alexey.tokarev@mail.ru 
responsible for resistance of the vascular bed (or parts of it) to the blood flow are a challenging task of utmost theoretical and practical importance.

Blood is rather a complex mixture, a colloid system composed of a carrier phase or plasma (aqueous solution of salts and proteins) and a dispersed phase or blood cells. These formed elements of blood are erythrocytes (red blood cells, RBCs), leukocytes (white blood cells), and platelets. Erythrocytes transport oxygen and carbon dioxide between lungs and capillaries; in this way, oxygen is delivered to body tissues and exchanged for their carbon dioxide. Erythrocytes comprise $40-45 \%$ of the blood volume whereas the remaining cells (platelets, leukocytes) account for approximately $1 \%$. For this reason, these are erythrocytes that almost wholly determine blood rheology [1-7] including its viscosity ${ }^{\dagger}$ and non-Newtonian properties. All rheological characteristics of blood are attributable to high concentration, marked deformability, and reversible aggregation of erythrocytes. The non-Newtonian effects are most readily apparent in the so-called microcirculation bed, i.e. blood vessels up to $\sim 100 \mu \mathrm{m}$ in diameter. In the macrocirculatory bed (i.e. larger vessels) they are less essential. Vessels of the microcirculation system (arterioles, capillaries, venules) are responsible for blood supply to all body tissues, and roughly $80 \%$ of the total blood pressure drop occurs in them. Erythrocytes traveling in the microcirculatory vessels exhibit a net migration towards the flow axis forming the so-called core of the flow and the cell-free nearwall region. It accounts for segregation of intravascular blood flow [7, 10-15]. The bulk of the core is composed of reversibly aggregated erythrocytes and the near-wall layer of plasma containing solitary erythrocytes, platelets, and other cells. This net displacement of erythrocytes from walls towards the flow axis causes the effective viscosity of blood to decrease, increases average cell velocity relative to that of blood, and diminishes hematocrit level in daughter branches compared with that in the parent vessel. These effects become more pronounced as the vessel diameter decreases. They are referred to as Fåhræus-Lindqvist effect, Fåhræus effect, and plasma skimming respectively $[6,8]$. In other words, non-uniform distribution of erythrocytes across the blood flow has a complicated influence on overall resistance of the microvasculature and effectiveness of its transport function. It accounts for the importance of its adequate description in mathematical simulations of blood flow.

Another consequence of non-uniform erythrocyte distribution across the bloodstream is the non-uniform distribution of other formed elements, platelets and leukocytes, that are forced by erythrocytes toward the vascular wall [16-18]. This fact is of great importance because both platelets and leukocytes must interact with the wall. Platelets are the main components of the hemostasis system forming protective plugs at sites of vascular damage, while leukocytes are cells of the immune system penetrating through the vascular wall to the sites of inflammation.

The present review is organized as follows. In Section 2, we consider the mechanism govern-

\footnotetext{
†There are several definitions of suspension viscosity [8]: apparent viscosity $\eta_{a}=\frac{\tau}{\dot{\gamma}}$ is determined in a rotational viscosimeter in which the shear rate $\dot{\gamma}$ is constant in space due to the linear velocity distribution; effective viscosity is measured using a capillary viscosimeter and calculated by the formula $\eta_{e f f}=-\frac{\pi R^{4}}{8 L} \frac{\Delta P}{Q}$, where $\Delta P$ is the pressure drop between the two ends of the tube of radius $R$ and length $L$, and $Q$ is the volume flow rate; dynamic viscosity $\eta$ enters the Navier-Stokes equations or its analogs as a proportionality factor between the double rate-of-strain tensor $d$ and the shear stress tensor $\Sigma$, see eq. (4.3). In the simplest case of a shear flow, the shear rate is a derivative of velocity perpendicular to the flow direction. In a general case it is defined by the second invariant of the strain rate tensor $D_{I I}=\sum d_{i j}^{2}$ as $\dot{\gamma}=\sqrt{2 D_{I I}}$ [9].
} 
ing initiation of migration of individual particles in the flow. It being directly related to particle deformability, the rigid and flexible particles are discussed separately. We then turn our attention to dense suspensions starting from the Lagrangian approach (Section 3) and further on to the Eulerian one (Section 4). In both cases, much attention is given to platelet migration in the presence of erythrocytes. In Section 5 we propose some new approaches to the erythrocyte lateral migration theory. In Section 6, we briefly discuss the main aspects of the mathematical analysis of the equations describing blood motion: the existence and uniqueness of their solution and its stability. Finally, Section 7 contains conclusions.

\section{Lateral migration of individual particles in the flow}

Hydrodynamic properties of platelets allow them to be considered as solids in diluted suspensions: (a) they rotate as solid spheroids and disks [5] and (b) congregate at a distance of $0.6 R$ from the tube axis [19]. Unlike platelets, erythrocytes are readily deformed and exhibit properties of both rigid and deformable particles depending on the shear rate (Section 2.2.1). For this reason, in what follows, we shall consider separately lateral migration of rigid and deformable particles. Everywhere in the paper rigid walls of the fluid conduit will be assumed although flexible wall may reinforce lateral migration due to the non-vanishing transversal fluid velocity.

\subsection{Single rigid particles and platelets}

2.1.1. The behaviour of rigid particles in a flow Observations have demonstrated that single rigid particles introduced into a flow moving in a long tube tend to an equilibrium position at the distance $R^{*} \approx 0.6 R$ from the axis, where $R$ is the tube radius. It is the so-called "tubular pinch effect" of Segré and Silberberg [20-27]. The rate of such lateral migration measured experimentally can be approximated by the equation [20]

$$
\frac{d r}{d t}=0.15 R e_{s} U \frac{r}{R}\left[1-\frac{r}{R^{*}}\right]
$$

where $r$ is the radial coordinate of the particle's centre, $R e_{s}$ is the particle Reynolds number ${ }^{\ddagger}$, and $U$ is the mean flow velocity. Results of thorough experiments indicate that the very possibility of lateral migration of rigid particles, its rate, and equilibrium position depend on many characteristics and properties of both the particles and the fluid. This phenomenon is observed only when the particle Reynolds number exceeds a certain threshold value $\left(R e_{s}>10^{-4} \div 10^{-3}\right)$ which suggests

\footnotetext{
${ }^{\ddagger}$ The tube Reynolds number is defined as $R e=\frac{2 R U}{\nu}$, where $\nu=\frac{\eta}{\rho}$ is a kinematic viscosity. The particle Reynolds number $R e_{s}$ (also frequently denoted as $R e_{p}$ ) is defined through the substitution of $U$ by the velocity of the particle relative to the undisturbed flow at the position of its centre $v_{s}=|v-u|$ (slip velocity) and of the tube radius $R$ by the particle radius $a: R e_{s}=\frac{2 a v_{s}}{\nu}$. Because at the flow axis $v_{s}=\frac{2}{3}\left(\frac{a}{R}\right)^{2} U_{m}[20,28-30]$ and in the tube $U_{m}=2 U, R e_{s}$ is sometimes found from the formula $R e_{s}=\frac{8}{3} a\left(\frac{a}{R}\right)^{2} \frac{U}{\nu}$. By analogy, the shear and rotational Reynolds numbers are defined as [26, 31, 32] $R e_{G}=\frac{4 a^{2} \dot{\gamma}}{\nu}, R e_{\Omega}=\frac{4 a^{2} \Omega}{\nu}$, where $\dot{\gamma}$ is the shear rate calculated at the centre of the sphere and $\Omega$ is its angular velocity.
} 
involvement of inertia in its development [23]. The equilibrium position shifts to the axis when the size of the particle increases or its rotation is hampered [24, 27] $\left(R e_{s}=1 \div 13\right)$. Experiments with particles of non-zero buoyancy in a vertical flow demonstrated that the relative velocity of the particles also affects the direction of its lateral movement: a particle moving faster than the flow migrates toward the wall while a slower moving one migrates to the axis [27]. At extremely high velocities of the flow ( $R e=30 \div 2000$ ), the equilibrium position is shifted towards the wall [23, 33 . At such $R e$ as well as in a pulsed flow, a few equilibrium ring zones may arise $[25,33]$. In a circular tube, the equilibrium position has a ring shape while four equilibrium positions in a tube of rectangular cross section are located on diagonals at equal distances from the centre [34]; in the Couette flow, the equilibrium position lies in the mid-plane [26].

The foregoing indicates the necessity of thorough consideration of the process of particle-flow interaction for the understanding of causes behind lateral migration. It is therefore opportune to discuss forces acting on a solitary particle in a viscous fluid flow depending on its translational and rotational velocity and on the proximity to the walls.

2.1.2. Lateral migration velocity of rigid particles Drag force acting on a rigid particle of radius $a$ in a steady state is $[31,35]$

$$
\bar{F}_{D}=-6 \pi a \eta \bar{v}_{s} \cdot\left(1+\frac{3}{8} \operatorname{Re}_{\mathrm{s}}+\mathrm{o}\left(\operatorname{Re}_{\mathrm{s}}\right)\right),
$$

where $\bar{v}_{s}=\bar{v}-\bar{u}$ is the relative velocity of the particle, $\bar{u}$ and $\bar{v}$ are the fluid and particle velocities, respectively. Given the non-uniform fluid velocity distribution, the Faxen force proportional to $\nabla^{2} \bar{u}$ is added to $\bar{F}_{D}$. Other additional terms are related to fluid acceleration, they are known as virtual mass term and history term (Basset force) [35].

Lateral forces acting on a rigid particle in a flow arise due to inertia of the fluid and the presence of the wall. Indeed, the lateral force vanishes upon linearization of the Navier-Stokes equations (cancellation of inertia terms) and in an infinite Poiseuille flow. However, at finite Re, the lateral force acting on the particle emerges. Its components are referred to as Magnus and Saffman forces.

Suppose a particle of radius $a$ rotates and has a non-zero speed with respect to the fluid flow. Then, its rotation will cause the fluid to accelerate on the one side and decelerate on the other. The pressure difference between the two sides arising in accordance with the Bernoulli law will create a "lift" force (Magnus force) directed normally to the particle relative velocity and axis of rotation. It equals $[31,35,36]$

$$
\bar{F}_{\text {Mag }}=\pi a^{3} \rho \cdot\left(\bar{\Omega} \otimes \bar{v}_{s}\right) \cdot\left(1+O\left(R e_{s}\right)\right) .
$$

The velocity of perpendicular movement due to this force can be found dividing by $6 \pi a \eta$ (equation (2.2)). Evidently, the Magnus force is unrelated to Re and fluid viscosity at low velocities; in other words, it describes interaction of the particle with the fluid layer immediately adjacent to its surface. Therefore, the Magnus force is essential only in hydro- and aerodynamic applications where particles and bodies are set in translational and rotational motion with respect to the carrier phase by some external action. 
If, however, the translational-rotational motion of the particle is caused by the shear movement of the surrounding viscous liquid, as in diluted suspensions, the situation becomes much more complicated [37]. In such a case, the particle affects the flow far apart (many diameters) from its surface, and the lateral force acting on it (Saffman force) results from inertia of the fluid in this wide region, unlike the Magnus force which has a local effect. In the simplest case (the linear velocity distribution in an infinite medium), the expression for the Saffman force has the form [31, $35,38]$

$$
F_{\text {Saff }}=3.22 a \eta v_{s} \sqrt{R e_{G}}=6.44 a^{2} v_{s} \sqrt{\rho \eta \dot{\gamma}}
$$

This formula is constrained by conditions $R e_{s}, R e_{G}<<1$ and $\varepsilon=\sqrt{R e_{G}} / R e_{s}>>1\left(v_{s}<<\right.$ $\sqrt{\dot{\gamma} \nu}$ ), i.e. by the smallness of inertial effects due to particle slipping (translational motion relative to the undisturbed fluid) compared with those produced by the shear (i.e., relative rotational motion). Generalization over the case of small $\varepsilon$ gives a factor $J_{1}(\varepsilon)$ in equation (2.4) that equals 1 at $\varepsilon \rightarrow \infty$, rapidly vanishes at $\varepsilon<1$, and assumes a weakly negative value at $\varepsilon<0.22$ [39]. It suggests a gradual decrease and even a change of direction of the lateral force when the particle's relative rotation slows down and slipping increases. Such dependence $F_{\text {Saff }}(\varepsilon)$ for $\varepsilon=0.3 \ldots 1.5$ and $R e_{s}<1$ was confirmed quantitatively in both theory [32] and experiment [40]. However, the change of the sign was not confirmed. Thus, on the whole, $J_{1}(\varepsilon)$ gives an S-shaped rise in the Saffman force upon a change of $\varepsilon$ from 0 to $\infty$. The final expression for the lateral force has the form [32]

$$
F_{\text {Lift }}=\operatorname{a\eta v}_{s}\left(3.22 J_{1}\left(\frac{\sqrt{R e_{G}}}{R e_{s}}\right) \cdot \sqrt{R e_{G}}-\frac{11 \pi}{32} R e_{G}+\frac{\pi}{4} R e_{\Omega}\right) .
$$

Here, the first term is the generalized force (2.4), the second - its correction of the next order, and the last one is the Magnus force (2.3). Numerical experiments [32] gave an idea of the relative contribution of the particle's rotational and sliding velocities to the lateral force: (1) a rise in the shear rate, i.e. increased $\varepsilon$, significantly enhances the lateral force, (2) the force is just as well retained in the absence of rotation $\left(R e_{\Omega}=0\right)$ while rotation appreciably amplifies it only at small $R e_{G}$, i.e. under conditions of derivation of the Magnus force equation.

The analysis performed by Saffman made it possible to explain many phenomena with the lateral migration of particles in diluted suspensions. The force (2.4) is directed towards the side where the streamline velocity is opposite to the particle's relative velocity. For a particle traveling in the Poiseuille flow, it means that the force is directed towards the axis when the particle lags behind the flow and toward the wall as it goes ahead of the flow. This situation is quantitatively consistent with the experimental results for particles with zero buoyancy. However, the Segré-Silberberg effect could be explained only if both the walls and the non-uniform shear rate gradient were taken into account. To this end, Cox and Brenner developed a general method for the computation of the force and the moment acting on a spherical particle placed in an arbitrary flow at a large distance $d$ from an isolated infinite flat wall or a cylinder wall $(R e<<a / d<<1)$; the particle was regarded as a point source of the force [37]. Application of this method to a particle in the Poiseuille flow [41] revealed the presence of three equilibrium positions of its lateral migration: two of them lying 0.62 of the half-width from the centre were stable whereas the central one was not. It fairly well 
explained the Segré-Silberberg effect. In the absence of the particle rotation, the stable equilibrium position proved to lie within 0.48 of the channel half-width from the centre, in agreement with the experimental observations of Oliver [24].

Cox and Hsu [42] used the same method to derive the expression for the lateral force acting on a spherical particle located at distance $d$ from the wall [43]:

$$
\bar{F}_{\text {Lift }}=a \eta v_{s} \cdot R e_{s} \cdot I
$$

where

$$
I \approx\left(0.56-\Lambda \frac{d}{a}+0.58 \Lambda^{2}\right) \pi
$$

$\Lambda=R e_{G} / R e_{p}=\dot{\gamma} a / v_{s}$. The value of coefficients in front of $\Lambda^{2}$ was found to slightly differ for a sphere with free and frozen rotation (0.58 compared with 0.57$)$ which suggests the negligible effect of the particle rotation on the lateral force. The formula (2.7) is inapplicable when the sphere touches the wall $(d \rightarrow a)$, and the separate consideration of this case gives $I=9.22$. The results of direct numerical calculations at varied $d / a$ ratio are in agreement with these estimates of $I$ [43]. Their approximation by polynomial in powers of $\Lambda$ and $d / a$ is used in modern Lagrangian models of single particle motion, such as designed to simulate platelet and monocyte motion in the blood flow for calculating the lateral force acting on them [44-45] (see Section 3.3).

\subsection{Single deformable particles and erythrocytes}

2.2.1. The behaviour of deformable particles in a flow The lateral migration of deformable particles (liquid droplets in emulsions, flexible rods and disks, RBCs and rouleaux), unlike that of rigid ones, occurs even at such low values of $\operatorname{Re}_{s}\left(<10^{-6}\right)$ where inertia of the fluid is negligibly small and rigid particles fail to migrate $[1,3,20,26,27,29,47]$. The velocity of this migration grows with increasing relative size and deformability of the particle, viscosity of the medium, and shear rate. The ability of a deformable particle to change its shape substantially complicates the task of determining the force acting on it because the shape of the particle is a part of the solution of the problem of its interaction with the external flow (as opposed to a rigid particle the shape of which remains unaltered). The mechanical properties of the particle are very important in the context of lateral migration because its shape is determined by the resultant of forces deforming it (external forces) and bringing it back to the equilibrium (surface and internal forces). It will be shown in this Section that erythrocytes exhibit properties of both rigid and deformable particles. It is therefore appropriate that their consideration should be preceded by that of a couple of special cases.

Droplets in emulsions have a spherical equilibrium shape due to surface tension forces. In a shear flow, their shape changes to an ellipsoid having the main axis directed at an angle to the flow while the droplet contents rotates around the centre of masses [1, 20, 29, 47, 48]. There are two origins of the lateral force acting on the droplet: its hydrodynamic interaction with the shear flow and with the wall [26]. In the absence of inertial effects, these forces vanish when the droplet 
resumes its symmetric shape as soon as it reaches the flow axis. In other words, the droplets in the Stokes regime of the Poiseuille flow migrate towards its axis as appears from both early analytical [20, 29, 47] and relatively recent numerical [49] models of droplet migration at equal phase densities. However, some analytical calculations predict droplet migration to the wall in a certain range of relative droplet-to-fluid densities $(0.5<\lambda<10)$ [26]. Numerical calculations for finite $R e$ values predict that droplets should migrate towards the axis only at $\lambda<1$ and small $R e_{s}$; while at $\lambda>1$ and small $R e_{s}$, and also at $R e_{s}>0.01(R e>5)$, the droplets must migrate off the axis thus exhibiting inertial effect [50].

As flexible rods and disks move in a shear flow they turn over with a periodically changing angular velocity and bend. Most of the time, their longitudinal axis remains parallel to the flow, then a rapid turn over the edge occurs. It is this property of flexibility that ensures their lateral migration towards the flow axis at a small Res whereas rigid particles of the same shape do not migrate under similar conditions [1, 20, 29, 47].

Vesicles have viscous contents encapsulated by an elastic inextensible membrane. The membrane prevents vesicle's stretching and imparts its rotation to the internal contents. The important difference of the behaviour of vesicles and droplets is that above a critical value of shear rate or viscosity ratio the tanktreading vesicle motion undergoes transition to the solid-like tumbling motion analogous to the rotation of rods and disks [48].

Erythrocytes resemble vesicles but have in addition a submembranous cytoskeleton that markedly enhances elasticity of the membrane and gives these cells a flattened biconcave shape. Thus, the erythrocytes' behaviour in a flow resembles in certain respects that of vesicles, deformable or rigid particles, depending on the conditions $[1,5,20,48]$. In a very slow flow where fluid inertia is negligibly small $\left(\dot{\gamma}<20 s^{-1}, R e_{s}<5 \cdot 10^{-5}\right)$, erythrocytes behave like rigid disks tumbling irregularly; their lateral migration is apparent only as movement from the wall, i.e. as depletion of the near-wall layer as thick as 1-2 erythrocyte diameters. Under these conditions, aldehyde-fixed (hardened) erythrocytes (HBCs) fail to migrate laterally thus exhibiting properties of rigid particles. At $\dot{\gamma} \sim 100 s^{-1}\left(R e_{s} \sim 10^{-4}\right)$ erythrocytes are oriented with respect to the flow direction and get somewhat extended under its influence. Their membranes move relative to the cell contents like tank threads but retain the biconcave shape. Such behaviour makes erythrocytes similar to vesicles and different from permanently rotating HBCs. In these conditions, the nearwall region is strongly and the axial zone is slightly depleted of erythrocytes; such situation may be interpreted as the onset of manifestation of the Segré-Silberberg effect. At $\dot{\gamma} \sim(1 \div 5) \cdot 10^{3} \mathrm{~s}^{-1}$ $\left(R e_{s}>10^{-3}\right)$ erythrocytes behave on the whole as solids; the Segré-Silberberg effect is well apparent as both near-wall and axial regions are depleted. Hardened erythrocytes migrate toward the equilibrium position $0.6 R$ from the axis while the equilibrium position of normal (deformable) erythrocytes is closer to the axis, $\sim 0.45 R$. The trajectory and the lateral migration velocity of HBCs are fairly well described by the equation (2.1); the velocity of RBCs lateral migration is 1-2 orders of magnitude higher than that of HBCs [20]. Thus, of the two effects (migration from the wall and from the axis) the former one is strongly exhibited by normal erythrocytes regardless of conditions whereas the latter is less apparent and limited to high shear rates. At all shear rates, deformability of erythrocytes significantly promotes their lateral migration although they always retain some rigidity which accounts for the qualitative difference of their behaviour from that of 
droplets.

Analytical and numerical models of individual droplet, vesicle, and erythrocyte dynamics in a tube make it possible to evaluate the role of particle deformation during lateral migration (see, for instance, [51, 52] and review [6]). Specifically, disturbances of near-axial equilibrium of a vesicle or an erythrocyte and the description of their interaction with the wall are debatable issues. The Lagrangian models developed for the description of the behaviour of both single erythrocytes and their suspension are considered in Section 3.2.

2.2.2. Lateral migration velocity of deformable particles Early theoretical works concerning the behaviour of droplets and elastic particles in a flow provided explanation for their experimentally observed migrations off the wall in the bounded flow and toward the axis in the Poiseuille flow [29, 30, 47, 53-55]. The velocity of lateral migration of a liquid droplet far from the wall estimated in [55] after the substitution of the radial coordinate by $R \cdot \dot{\gamma} / \dot{\gamma}_{w}$ ( $\mathrm{R}$ is the tube radius) and simple algebraic transformations can be written in the form

$$
\bar{V}_{\text {drop }}=C \cdot a^{2} k \cdot \bar{n},
$$

where $C=\eta a \dot{\gamma} / \sigma$ is the capillary number, $k=\frac{d \dot{\gamma}}{d y}$ is the shear rate gradient (assumed to be constant), and $\bar{n}$ is the normal to the flow directed toward the axis. The expression for the migration velocity near the wall has the form $[26,56,57]$

$$
\bar{V}_{\text {drop }}=a \dot{\gamma} \cdot\left(\frac{a}{h}\right)^{2} D \cdot f(\lambda) \cdot \bar{n},
$$

where $a$ is the radius of an undistorted droplet, $h$ is the distance between its centre and the wall, $D=\frac{L-B}{L+B}=C \cdot \frac{19 \lambda+16}{16 \lambda+16}$ is the deformation of the droplet ( $\mathrm{L}$ and $\mathrm{B}$ are the lengths of the principal axes if the droplet's shape is approximated by a prolate ellipsoid), $C=\eta a \dot{\gamma} / \sigma$ is the capillary number, $\sigma$ is the interfacial tension, $\lambda=\eta_{1} / \eta$ is the ratio of droplet to carrier phase viscosities, and the values of function $f(\lambda)$ for any $\lambda$ fall within the range from 0.55 to 0.58 . These formulas are valid for the Stokesian regime and small deformations.

Although the formula (2.9) was derived to describe the migration of a single droplet, certain authors used it in an attempt to explain the concentration profiles in diluted and concentrated suspensions with low viscosity ratio $[58,59]$. To this effect, they considered the balance of flux $V \cdot \Phi$ (with the explicit dependence $V_{\text {drop }}(y)$ additively taking into account both walls) and shear-induced diffusion (with diffusion coefficient $D_{\text {eff }} \sim \dot{\gamma} a^{2} \Phi$ ), analogous to the equation (4.28). A similar approach was employed in [60] for an erythrocyte suspension. However, its applicability to a concentrated suspension (in contrast to diluted one) appears questionable because it ignores screening of the droplet-wall interaction by other (surrounding) droplets.

Vesicles like droplets assume a roughly similar ellipsoid shape in the shear flow. The first estimation of lateral migration velocity of a vesicle was obtained on the assumption that it had an ellipsoid shape with semi-axes $a_{1,2,3}$ ( $a_{3}$ being the major semi-axis) of known lengths and that the membrane velocity linearly depended on coordinates (i.e. the condition of incompressibility and inextensibility was not fulfilled). It yielded [61] 


$$
V_{v e s}=U\left(\lambda, \frac{a_{1,2}}{a_{3}}\right) \cdot a \dot{\gamma} \cdot\left(\frac{a}{h}\right)^{2} .
$$

This formula is analogous to (2.9) at $D=$ const in that it gives $V \sim \frac{a^{3}}{h^{2}}$. The velocity amplitudes are compatible too because the values of function $U\left(\lambda, \frac{a_{1,2}}{a_{3}}\right)$ found numerically by parameter variation fell within a range of $0 \div 0.5$. However, the formula (2.9) predicts the quadratic dependence $V_{\text {drop }}(\dot{\gamma})$ since $D \sim C \sim \dot{\gamma}$ whereas the equation (2.10) can not by itself predict the dependence $V_{\text {ves }}(\dot{\gamma})$ because the vesicle shape $\left(\frac{a_{1,2}}{a_{3}}\right.$ ratios) is assumed to be already known. Moreover, the dependence of $U$ on the parameters of ellipsoid shape being rather strong [61], it appears necessary to further develop this theory to be able to evaluate the degree of vesicle and erythrocyte deformation $D$ depending on the shear rate. Then, it will be possible to obtain the explicit dependence of erythrocyte migration velocity on the shear rate $V_{R B C}(\dot{\gamma})$. For the extreme cases of $D$ and $U$ independence of $\dot{\gamma}$ and their linear dependence on it, the dependence $V(\dot{\gamma})$ described by these two formulas is linear and quadratic, respectively. Hence, one may expect a final dependence $V_{R B C}(\dot{\gamma})$ intermediate between $\dot{\gamma}$ and $\dot{\gamma}^{2}$.

Experimental and numerical data are insufficient to draw the unambiguous conclusion about the character of dependence $V_{R B C}(\dot{\gamma})$. For example, the assumption of its linearity (i.e. constant erythrocyte deformation) and the choice of $U=0.01$ make it possible to quantitatively describe the degree of thickening of the RBC-free near-wall layer observed in an in vitro experiment during the blood passage through stenosis [62]. Estimation of the erythrocyte lateral migration velocity near the wall from the onset of hemolysis during vacuum plasma suction through the porous wall yielded a strictly quadratic dependence [63]. Unfortunately, it was but a preliminary report (only two experimental points) and the paper itself is unavailable. Numerical calculations demonstrate the saturating dependence of vesicle deformation on the local shear stress $[64,65]$. This case can be regarded as an intermediate one because the dependence $V_{R B C}(\dot{\gamma})$ must be quadratic at small $\dot{\gamma}$ with gradual power reduction parallel to the growth of $\dot{\gamma}$.

2.3. Transition from diluted to dense suspensions and blood As shown in the foregoing, the interaction of a single particle with a shear flow may result in its lateral migration that becomes markedly enhanced if the particle is deformable. However, as soon as the volume fraction of the particles amounts to $6 \%$, the mean distance between their surfaces proves to be smaller than the diameter [35] and particle-particle interaction begins to prevail. Studies of concentrated suspensions of rigid particles (see Sections 3.1 and 4.2) have ultimately shown that particle-to-particle collisions in themselves may lead to the strongly non-uniform distribution of particles across the flow. Therefore, we now pass to the consideration of concentrated suspensions, in the first place blood.

A distinctive feature of erythrocytes, apart from their mechanical properties, is the ability to aggregate into "rouleaux". A single rouleau containing several erythrocytes migrates across the flow more efficaciously than an isolated erythrocyte $[5,20]$ by virtue of its high flexibility due to deformability of erythrocytes and their weak binding to each other. The core of the blood flow at low shear rates is formed by a continuously rearranging conglomerate of such rouleaux. With a rise in the shear rate (within a range of 8.8-123 $\mathrm{s}^{-1}$ ) [5] as a result of rouleaux disruption, 
the core broadens while the RBC-free near-wall layer accordingly becomes thinner. Inhibition of erythrocyte aggregation has similar effect. As the shear rate increases further (in a range 240-1200 $s^{-1}$ ) [19], the opposite effect takes place; that is, the lateral migration of erythrocytes causes the near-wall layer to broaden. Thus, the aggregation of erythrocytes exerts a strong influence on both blood rheological properties and the displacement of other blood cells toward the walls.

Unlike erythrocytes, platelets are numerically insignificant, fail to aggregate (in the absence of stimulation), and appear to be practically rigid; their linear dimension is 3-4 times smaller than that of erythrocytes. On the other hand, erythrocytes have a very strong effect on the motion of platelets in the blood forcing them to the periphery of the flow, into the near-wall layer. For this reason, the description of the platelet motion in the absence of erythrocytes seems to be a simple task whereas its description in blood requires the erythrocyte-platelet interaction to be somehow taken into consideration. The inverse effect (that of platelets on erythrocytes and the flow) is practically non-existent (in the absence of platelet aggregation); in other words, the one-way coupling takes place. Both Lagrangian and Euler methods are extensively used in theoretical studies of erythrocyte and platelet dynamics.

\section{Description of suspension Segregation by the Lagrangian methods}

Methods of this class are designed to study the dynamics of individual particles in suspensions. They have been developed relatively recently, after large amounts of the computational power became available, but already brought success in the description of non-uniform particle distribution across the flow and related rheological effects.

3.1. Suspensions of rigid particles In Stokesian dynamics [66, 67], the acceleration of each particle is governed by Newton's second law depending on the sum of forces acting on it from the neighboring particles and the walls. The numerical calculations fairly well describe steady-state non-uniform concentration profiles and flattening of velocity profiles observed in experiments. The resulting particle redistribution rate across the flow is consistent with the theoretically and experimentally derived dependence $D \sim \dot{\gamma} a^{2}$. The results of such direct calculations are considered together with the experimental findings and compared with the balance models (see Section 4.2.2). Moreover, they allow for experimentally impracticable assessments, e.g. evaluation of the influence of particle roughness on their lateral migration [66].

3.2. Erythrocytes In the discrete element (or particle) method (DEM, DPD) matter is taken in the form of single particles moving freely and interacting with one another according to some definite rules. The correct choice of these rules ensures the behaviour of the medium in conformity with the Navier-Stokes equations and the behaviour of deformable particles in agreement with flexibility laws. In [68], blood plasma and platelets were regarded as separate particles and erythrocytes as a closed chain of bound particles (in 2D). This model gives the shape of the deformed erythrocyte 
for the case of a single cell traveling in a capillary and results in their net migration toward the axis for the case of suspension moving in a larger vessel.

The lattice Boltzmann method (LBM) was introduced into hydrodynamics from gas dynamics and suits well to simulate incompressible media [69]. In this method, matter is given as a lattice, with particles jumping between its nearest-neighbour sites with a certain probability. The adequate choice of the jumping rules ensures a good agreement of the results obtained by LBM with the exact solutions of the Navier-Stokes equations. This method is successfully employed to simulate 2D and 3D blood dynamics and to describe the motion of individual erythrocytes and their concentrated suspensions as well as the influence of erythrocytes on leukocytes and platelets [70-72] (see review [34]). The immersed boundary method (IBM) proved highly efficacious for the simulation of deformable particles, such as erythrocytes [73]. It makes possible simulation of their pair aggregation [74]. The most efficient is a combination of IBM and LBM [71] that was used to simulate even the formation of rouleaux in the flow [75]. The aggregation is introduced phenomenologically through the Morse intermembrane potential. The models with deformable erythrocytes fairly well describe the depletion of erythrocytes in the near-wall layer, the Fåhræus and Fåhræus-Lindqvist effects $[71,73,76]$. The in-depth investigation of erythrocyte aggregation mechanics in the absence of a flow is possible by different methods [77].

3.3. Platelets Classical realization of the Lagrangian method is based on the integration of the motion equations for each single platelet $[44-46,78,79]$. The platelet velocity may be assumed to be equal to the medium velocity (solutions of the Navier-Stokes equations $(4.2-4.5)$ ) $[78,79]$ or computed taking account of the forces (2.2) and (2.5) [44-46]. The discrete element method (DEM, DPD) mentioned in Section 3.2 provides for an essentially different description of the fluid. In this method, both platelets and plasma are simulated by single rigid particles [68, 8983]. Most of these studies describe platelet aggregation in the absence of erythrocytes, i.e. on the assumption of the uniform platelet distribution. Studies $[80,82]$ are the exceptions in that the platelet migration to the near-wall region under effect of erythrocytes was simulated by the application of a phenomenologically given external force. The direct method for the simulation of the non-uniform platelet distribution might consist of taking into account the presence of both erythrocytes and platelets in blood as described in [68], but such studies were never reported. None of the above works was designed to reproduce the Segré-Silberberg effect. As mentioned in Section 3.2, the application of LBM allowed all major platelet-related effects to be described; viz. platelet displacement from the core by erythrocytes and the Segré-Silberberg effect. The Level set method also appeared quite applicable to the description of erythrocyte migration towards the periaxial region with platelet displacement towards the wall [84].

\section{Continuous approach to the description of suspension segre- gation}

In methods of this class, as opposed to the preceding ones, suspensions are treated as continuous media with the locally-averaged properties. In single-phase models, the suspension behaviour is 
given by the equations for 1) the whole suspension and 2) the dispersed phase.

1) The equations for the whole suspension are the continuity equation

$$
\frac{\partial \rho}{\partial t}+\nabla \cdot(\rho \bar{u})=0
$$

and the momentum balance equation

$$
\frac{D \bar{u}}{D t}=\frac{1}{\rho} \nabla \Sigma+\bar{F} .
$$

Here, $\rho$ is density, $\bar{u}$ - the fluid velocity vector, $\Sigma-$ the deformation stress tensor (the Cauchy stress tensor), $\bar{F}$ - the external volume force (e.g. gravity force), and $\frac{D}{D t}=\frac{\partial}{\partial t}+(\bar{u} \cdot \nabla)$ is the material derivative $[9,85,86]$. The constraint equation $\Sigma=\Sigma(\cdot)$ known as the rheological equation is essentially dependent on the properties of the carrier and disperse phases. In the case of a viscous incompressible medium, the rheological equation is represented by generalized Newton's law

$$
\Sigma=-p I+2 \eta d
$$

where $p$ is pressure, $I$ - the unit tensor, $\eta$ - dynamic viscosity, $d$ - the rate-of-strain tensor

$$
d=\frac{1}{2}\left(\nabla \bar{u}+(\nabla \bar{u})^{T}\right) .
$$

The quantity $2 \mu d$ is the so-called extra-stress tensor

$$
S=2 \eta d=\eta \cdot\left(\nabla \bar{u}+(\nabla \bar{u})^{T}\right) .
$$

Incompressibility of the medium and its constant density account for the following form of the continuity equation

$$
\nabla \cdot \bar{u}=0 .
$$

The resulting equations (4.2-4.5) are called the Navier-Stokes equations. The boundary conditions are usually the no-slip conditions $(\bar{u}=0)$ and the constant inflow/outflow velocity or the pressure given at different parts of the boundary. Normally, various simplifications of these equations are used as listed below.

First, in the majority of the problems, blood motion under the action of external forces can be neglected assuming that $\bar{F}=0$. Second, it is possible to confine the consideration to a steady-state solution. In this case, the momentum balance takes the form

$$
\nabla \Sigma=0
$$

Third, in the case of a flow parallel to the $x$-axis, this expression reduces to the equation in the $y$-direction:

$$
\frac{d}{d y}\left(\eta(\Phi) u_{y}^{\prime}\right)=p_{x}^{\prime}
$$


where $p_{x}^{\prime}$ is the known pressure gradient.

In the framework of the continuous approach, specific properties of a suspension are determined by its dynamic viscosity. For a Newtonian medium (water, blood plasma), dynamic viscosity is a constant coinciding with apparent and effective viscosity; therefore, the above equations are sufficient to describe all macroscopic properties of the fluid. However, the experimentally measured viscosity of non-Newtonian or rheological media is a complicated function of the shear rate, its time derivatives, etc. and may depend on the measurement method, e.g. the geometry of the viscosimeter. In the case of particles suspended in a Newtonian fluid, it may be a consequence of deformation, aggregation or non-uniform distribution of the particles. These factors jointly affect the blood (see Section 2.3). While deformation and aggregation of erythrocytes can be described in the framework of the rheological models (Section 4.1.1), the consideration of their non-uniform distribution requires the introduction of an additional equation.

2) The equation for particle motion in suspensions can be written in the form

$$
\frac{D \Phi}{D t}=-\nabla \bar{J}
$$

where $\Phi$ is the particle volume fraction and $\bar{J}$ is the flux arising from the difference between the mean particle and suspension velocities:

$$
\bar{J}=\Phi \cdot\left(\left\langle\bar{u}_{p}\right\rangle-\bar{u}\right) .
$$

The form of the equation (4.9) depends on the anticipated mechanism of interaction between the particles and the carrier phase (Sections 4.1.3, 4.1.4, 4.2.1, 4.2.2, and 4.3). Alternatively, a separate Navier-Stokes equation for the dispersed phase may be written out (Section 4.1.5).

4.1. Dense suspensions of deformable particles and erythrocytes The unique blood properties determined by deformability and aggregability of erythrocytes are apparent even in the case of their uniform distribution. The aggregation leads to a sharp increase of apparent viscosity as the shear rate decreases below $\sim 50 s^{-1}$ while deformation causes reduction of viscosity with the growing shear rate. A variety of empirical dependences of apparent viscosity on the shear rate, hematocrit, and the degree of erythrocyte aggregation have been proposed to take these effects into consideration. However, it proved necessary to take account of the non-uniform erythrocyte distribution across the flow for a non-contradictory explanation of blood anomalies which manifest themselves during its movement in tubes and blood vessels and increase as their diameter decreases (Fåhræus, Fåhræus-Lindqvist effects, plasma skimming effects).

4.1.1. Rheological models of blood The most widely known rheological models of blood (power-law, Carreau, Casson, and Quemada models) [6, 9, 87-89] were developed empirically to describe the results of measurements of blood apparent viscosity in rotational viscosimeters; they disregard the non-uniformity of erythrocyte distribution. The apparent viscosity is assumed to depend on the shear rate according to one or another law whereas the parameters are the functions of hematocrit. In the power-law model, a rise in viscosity with decreasing shear rate is described in the simplest way: 


$$
\eta_{a}=k \dot{\gamma}^{n-1}
$$

where $k$ and $n(n<1)$ are the parameters.

In the Carreau model $[9,87,88,90-93]$, the range of viscosity variations is restricted by the limiting values of $\eta_{0}$ (viscosity at rest) and $\eta_{\infty}$ (asymptotic viscosity at $\dot{\gamma} \rightarrow \infty$ ):

$$
\eta_{a}=\eta_{\infty}+\left(\eta_{0}-\eta_{\infty}\right)\left(1+(\lambda \dot{\gamma})^{a}\right)^{\frac{n-1}{a}}
$$

Here, $a$ and $\lambda$ are the parameters.

The Ree-Eyring model is based on the notion of dense suspension movements as jumps of its particles between adjoining cells separated by the energy barrier. The shear rate is assumed to be proportional to the difference between jump frequencies along and counter flow and the height of the energy barrier in these directions to be dependent on the shear stress [94]. Two kinds of rearrangement processes are introduced for erythrocytes; namely, the slow aggregation with the characteristic time $t_{1}$ and rapid deformation/reorientation with the characteristic time $t_{2}$ [95]. These two processes determine blood viscosity at low and high shear rates, respectively. Apparent viscosity turns out to be a shear rate function:

$$
\eta_{a}=\eta_{\infty}+A_{1} \frac{t_{1} \dot{\gamma}}{\sinh ^{-1}\left(t_{1} \dot{\gamma}\right)}+A_{2} \frac{t_{2} \dot{\gamma}}{\sinh ^{-1}\left(t_{2} \dot{\gamma}\right)}
$$

Here $A_{1}$ and $A_{2}$ are the amplitudes of viscosity variation under the action of the two processes.

The Casson model suggests the linear dependence of $\sqrt{\tau}$ on $\sqrt{\dot{\gamma}}$ observable in experiment at $\dot{\gamma}>2 s^{-1}$ :

or

$$
\sqrt{\eta_{a}}=\sqrt{\eta_{\infty}}+\sqrt{\frac{\tau_{0}}{\dot{\gamma}}}
$$

$$
\begin{array}{cc}
\sqrt{\tau}=\sqrt{\eta_{\infty} \dot{\gamma}}+\sqrt{\tau_{0}} & \left(\tau \geq \tau_{0}\right) \\
\dot{\gamma}=0 & \left(\tau<\tau_{0}\right)
\end{array},
$$

where $\tau_{0}$ is the yield stress. The physical meaning of $\tau_{0}$ is that the force applied at $\tau<\tau_{0}$ is insufficient to rearrange aggregated particles in a suspension. This model is inadequate at small $\dot{\gamma}$ because the experimental dependence of $\sqrt{\tau}$ on $\sqrt{\dot{\gamma}}$ deviates from the straight line and tends to zero at $\dot{\gamma} \rightarrow 0$. It is impossible to reliably measure the yield stress by the traditional methods (in a viscosimeter) due to the depletion of erythrocytes and their sedimentation in the near-wall layer; the very existence of yield stress is questioned by certain authors. A serious drawback of the Casson model from the mathematical point of view is the fact that $\eta_{a} \rightarrow \infty$ at $\dot{\gamma} \rightarrow 0$. It is possible to correct by introducing the constant $\sqrt{\Lambda}$ into the denominator of the viscosity equation [96, 97]:

$$
\sqrt{\eta_{a}}=\sqrt{\eta_{\infty}}+\frac{\sqrt{\tau_{0}}}{\sqrt{\Lambda}+\sqrt{\dot{\gamma}}}
$$


The resulting model is equivalent to the Quemada model considered below at constant hematocrit and rapid aggregation [97-99].

Quemada's model is designed to take account of particle aggregation in concentrated suspensions of different nature [89, 100-102]. Its application to blood is based on assigning viscosity, by analogy with concentrated colloidal suspensions, in the form

$$
\frac{\eta_{a}}{\eta_{P}}=\left(1-\frac{k \Phi}{2}\right)^{-2}
$$

where $\Phi$ is the erythrocytes volume fraction (hematocrit/100\%), $k$ is the phenomenological structure factor regarded as the effective internal viscosity of the particles, $k \Phi<2$. The kinetic equation for $k$ has the form

$$
\frac{\partial k}{\partial t}+(\bar{V} \cdot \nabla) k=\frac{1}{\tau_{A}}\left[k_{0}-k-\sqrt{\dot{\gamma} / \dot{\gamma}_{C}}\left(k-k_{\infty}\right)\right] .
$$

In the steady-state conditions it reduces to

$$
k=\frac{k_{0}+k_{\infty} \sqrt{\dot{\gamma}_{/ \dot{\gamma}_{C}}}}{1+\sqrt{\dot{\gamma} / \dot{\gamma}_{C}}} .
$$

Here, $k_{0}$ and $k_{\infty}$ are the limiting $k$-values, $\dot{\gamma}_{C}$ is the critical shear rate, $\tau_{A}$ - characteristic aggregation time. The hematocrit dependence of the parameters was evaluated in [89, 96, 98, 103-105]. This model is fairly well verified and, unlike the previous ones, has a good physical basis.

Rate-type viscoelastic Oldroyd models have the "history" terms [87, 167]:

$$
S+\lambda_{1} \stackrel{\nabla}{S}=2 \eta_{0}\left(d+\lambda_{2} \stackrel{\nabla}{d}\right)
$$

Here, $\eta_{0}$ is a zero shear rate viscosity, $\lambda_{1}$ and $\lambda_{2}$ are relaxation time constants $\left(0 \leq \lambda_{2}<\lambda_{1}\right.$, $\nabla$ stands for the upper-convected derivative: $\stackrel{\nabla}{S}=\frac{D S}{D t}-S \cdot \nabla u-(\nabla u)^{T} \cdot \mathrm{S}$. In particular, the Oldroyd-B model is based on the decomposition of the total extra-stress tensor on non-Newtonian (polymeric, dispersed phase, ect.) and Newtonian (solvent) parts: $S=S_{1}+S_{2}$,

$$
\begin{gathered}
S_{1}+\lambda_{1} \stackrel{\nabla}{S_{1}}=2 \eta_{1} d \\
S_{2}=2 \eta_{2} d,
\end{gathered}
$$

where $\eta_{1}+\eta_{2}=\eta_{0}, \frac{\eta_{2}}{\eta_{1}+\eta_{2}} \lambda_{1}=\lambda_{2}$. This relation under simplifying hypotheses (for instance, independence of the viscosity and of the strain rate $d$ ) leads to the following system of equations:

$$
\begin{gathered}
\frac{D \bar{u}}{D t}=\frac{1}{\rho}\left(\eta_{2} \Delta \bar{u}-\nabla p+\nabla \cdot S_{1}\right)+\bar{F} \\
\nabla \cdot \bar{u}=0
\end{gathered}
$$




$$
S_{1}+\lambda_{1}\left(\frac{D S_{1}}{D t}-S_{1} \cdot(\nabla \bar{u})^{T}-\nabla \bar{u} \cdot S_{1}\right)=2 \eta_{1} d
$$

This system of equations should be equipped with the initial condition for both $\bar{u}$ and $S$. In the case of the boundary value problem, the Dirichlet type boundary condition is set for $\bar{u}$ only. In [167] the theorems on existence of solution and its stability are formulated for a domain with $C^{3}$ smooth boundary. The advantage of the Oldroyd-B model is that it combines the properties of the parabolic Navier-Stokes equations with the hyperbolic transport-like vector-valued equation. And one more class of blood rheological models - micropolar fluids - will be referred to in Section 4.1.3 as it gave some progress in accounting for the effect of non-uniform erythrocyte distribution.

4.1.2. The prescribed distribution of erythrocytes When the blood flow is examined under the near-physiological conditions (in a tube), the effective viscosity being measured proves to depend on the tube radius (the Fåhræus-Lindqvist effect) [8, 89]. As the diameter diminishes to below $500 \mu \mathrm{m}$, the viscosity begins to lower and reaches the minimal value ( 3 times smaller than the initial one) when the tube diameter becomes roughly equal to the erythrocyte diameter $(\sim 7 \mu \mathrm{m})$; as the tube diameter decreases further, the viscosity rises very steeply. These changes are a direct result of the non-uniform distribution of erythrocytes across the flow. Therefore, the next logical step in the description of such non-uniform distribution is the assumption of uniform distribution of erythrocytes in the flow core and their complete absence in the $\delta$-thick near-wall layer. This layer is considered to be a Newtonian liquid and a number of rheological laws are assigned in the core, viz. Newton's [106, 107], Casson's [108], and Quemada's [104] laws. Alternatively, the core is regarded as quasi-solid (with constant velocity) [104]. The thickness of the near-wall layer in such models is a variable parameter that can be adjusted to describe the velocity profile and the Fåhræus-Lindqvist effect. Due to high sensitivity to $\delta$, such models are mostly used for the qualitative explanation of the above effects. An interesting extension of the idea of representing the core of the flow and its near-wall layer as two immiscible liquids is the dynamic definition of the boundary by the Level set function method [98, 109]. It allows for the simulation of the blood flow in a region with complicated geometry. Note, that the resulting thickness of the near-wall layer is 1-2 erythrocyte diameters, so the models in question are on the verge of applicability of the Euler approach and even go beyond it; hence, the necessity of the cautious interpretation of the results obtained with their help.

The real distribution of erythrocytes across the flow is far from stepwise; in contrast, their concentration grows monotonically from the vessel wall to the axis as shown both in vitro $[13,19]$ and in vivo [11- 15]. The direct assignment of this distribution by an analytical formula leads to the models with spatially distributed coefficients of the rheological equation. Different versions of such assignment in the analytical form were proposed. The simplest ones are parabolic [14], piecewise-linear and piecewise-parabolic [110]. A smoother profile is obtained when a high-degree $(n=20)$ polynomial is used $[96,111]$ :

$$
\Phi=\left\{\begin{array}{cc}
\Phi_{\min } & \lambda \leq r_{1} \leq 1 \\
\Phi_{c}\left(r_{1}\right) & r_{1}<\lambda
\end{array}\right.
$$




$$
\begin{gathered}
\Phi_{c}\left(r_{1}\right)=\Phi_{\max }-\left(\Phi_{\max }-\Phi_{\min }\right) \times \\
{\left[\frac{n(n-1)}{2}\left(\frac{r_{1}}{\lambda}\right)^{n-2}-n(n-2)\left(\frac{r_{1}}{\lambda}\right)^{n-1}+\frac{(n-1)(n-2)}{2}\left(\frac{r_{1}}{\lambda}\right)^{n}\right]} \\
\Phi_{\max }=\Phi_{\min }+\left(\Phi_{0}-\Phi_{\min }\right) \frac{(n+1)(n+2)}{(n-1)(n-2) \lambda^{2}} .
\end{gathered}
$$

Here, $\lambda=0.5 \div 1$ is the core radius and $0 \leq r_{1} \leq 1$ is the distance from its axis, both normalized to the tube radius; $\Phi_{\min }$ is the volume fraction of erythrocytes in the near-wall layer. Coefficients of the polynomial are chosen such as to maintain the given average volume fraction $\Phi_{0}$ in the tube (the integral of $\Phi \cdot r d r$ ). Because the coincidence between core and tube axes is not required in this method, it permits not only to calculate the velocity profile but also to study the effect of asymmetric erythrocyte distribution which is strongly pronounced and versatile in vivo [8, 14, 112]. The distributions of the erythrocyte volume fraction at different hematocrit levels observed in long tubes under in vitro experimental conditions [19] can be more exactly interpolated in the form

$$
\begin{gathered}
\Phi_{R B C}(r)=\Phi_{m} \cdot\left[1-\exp \left(a-b \Phi_{m}\right)+\exp \left(a-b \Phi_{m} \cdot\left(1-r_{1}^{2}\right)\right)\right]^{-1} \\
\Phi_{m}\left(\Phi_{D}\right)=\frac{1.45 \Phi_{D}}{0.5+\Phi_{D}} .
\end{gathered}
$$

Here, $\Phi_{m}$ is the erythrocyte volume fraction at the flow axis, $\Phi_{D}$ - the inflow erythrocyte volume fraction (discharge hematocrit/100\%), $r_{1}$ is the radial coordinate normalized to the tube radius. The values of constants $a$ and $b$ are fitted as $a=3.5$ and $b=9$. Direct assignment of the erythrocyte distribution leads to the mathematical models of the blood flow consistent with the results of different experiment. However, these are the non-closed models because the solution of the problem strongly depends on the given erythrocyte distribution. In what follows, we pass to the Eulerian models in which the erythrocyte distribution is a part of the solution of the complete problem.

4.1.3. Micropolar and micromorphic models The theory of micropolar fluids (MPF) [113-115] prescribes to each element of the medium a micro-rotational inertia, in addition to its mass. Therefore, the continuity and momentum balance equations (4.1), (4.2) are supplemented by the related equations for conservation of the amount of micro-rotation $\bar{G}$. In the steady state the resulting set of equations for an incompressible fluid with constant density and viscosity has the form

$$
\begin{aligned}
& \nabla \cdot \bar{u}=0 \\
& (\eta+k) \nabla^{2} \bar{u}+k \nabla \otimes \bar{G}=\nabla p \\
& \gamma \nabla^{2} \bar{G}+k \nabla \otimes \bar{u}-2 k \bar{G}=0
\end{aligned}
$$

Here, $k$ is the vortex viscosity coefficient, $\gamma$ is the spin gradient viscosity coefficient. A more general class is represented by micromorphic fluid models (MMF) that take into account microdeformations of fluid particles, besides their microrotation $[115,116]$. The resulting system of equations 
for the velocity of movement and rotation constituted the basis of many blood flow models (see reviews $[117,118]$. Because they disregarded the non-uniform distribution of erythrocytes, their predictions were in conflict with the known facts about the blood flow in the microvessels, such as the flattened velocity profile, the Fåhræus-Lindqvist effect, etc. [3, 119, 120]. Nevertheless, these models proved applicable to the study of macrocirculatory vessels [121, 122]. For this reason, in many subsequent investigations, as well as in most of the aforementioned rheological models, the blood was considered as two immiscible fluids: the core of the flow with a fixed width being a micropolar fluid and the near-wall layer being a Newtonian one [123, 124] (see the review in book [125]).

There are at least two modifications of the micropolar theory predicting the profile of the erythrocyte concentration distribution. One of them is based on the assumption that particles are distributed over the space so that they exert the minimal mutual influence on the viscous energy dissipation rate in the microvolume surrounding each of them. It leads to the algebraic dependence of the local particle concentration $c$ on the empirical scalar "measure of rotation" with respect to the medium $R$ [126]:

$$
c=\alpha_{1}-\alpha_{2} R^{2}
$$

The other modification of the micropolar theory postulates a linear dependence of the gradient of particles' chemical potential, $Z$, on their rotational velocity [127-129]:

$$
-\nabla Z=\bar{J} / \alpha_{1}-\alpha_{2} \bar{J} \times \bar{B}-\alpha_{3} \operatorname{rot} \bar{\omega}+\alpha_{4} \operatorname{rot} \bar{\omega} \times \bar{B},
$$

where the symmetric part of tensor $\bar{\omega}$ is the average rate of strain within the particles contained in an elementary volume of suspension, the skew symmetric parts of tensors $\bar{\omega}$ and $\bar{B}=\bar{\omega}-\bar{\Omega}$ are the absolute and relative particle spin velocities, respectively, $\bar{\Omega}=\frac{1}{2}$ rot $\bar{v}$ is the tensor of medium spin velocities, $\alpha_{1}$ and $\alpha_{2}$ are the constants, $\alpha_{3}$ and $\alpha_{4}$ - the functions of $\Phi$. In the one-dimensional case, the system of equations was reduced to 2 coupled equations: for particles' rotational velocity and concentration. In both approaches, the choice of parameters permits to describe many specific features of the blood movement, such as the Segré-Silberberg effect at $\Phi \rightarrow 0$ (which corresponds to the platelet behaviour in the absence of erythrocytes), the marked depletion of the near-wall layer in a concentrated suspension (corresponding to the behaviour of erythrocytes), the Fåhræus and Fåhræus-Lindqvist effects, etc. Neither approach received any further development [130, 131], in the first place because of a large number of variables (components of particle and medium rotation tensors) and unknown constants and because of the difficulty to choose the boundary conditions for spin velocity $[132,133]$.

4.1.4. Blood fluctuation temperature One more serious disadvantage of the micropolar models is the overestimation of the degree of ordering of erythrocyte rotation in the flow. The thorough microscopic studies of rheological properties of concentrated erythrocyte ghost suspensions demonstrated that their rotation is hampered substantially by continuous collisions between the cells, their deformation and aggregation [5, 134]. These findings account for the shift of emphasis 
in the concept of particles' micromovements from rotation to dispersive motion [106, 135] resulting from continuous collisions. This motion can be quantitatively characterized by the coefficient of dispersion calculated, by analogy with the coefficient of thermal diffusion of the molecules, as the ratio of mean square lateral motion of the particle to double time between observations [4]:

$$
D=(\Delta r)^{2} / 2 \Delta t
$$

At normal for in vivo circulation shear rates, $D$ is 2-3 orders of magnitude higher than the coefficients of erythrocyte thermal diffusion derived from the Stokes-Einstein equation

$$
D_{B r}=k_{T} T / 6 \pi \eta a .
$$

where $T_{T}$ is the absolute temperature and $k$ is the Boltzmann constant. By analogy to this formula the hydrodynamic (fluctuation) temperature of suspension $T_{F}$ can be defined by writing [135]

$$
D=k T_{F} / 6 \pi \eta a
$$

The value of $T_{F}$ thus computed proves to be on the order of $10^{4}-10^{5} \mathrm{~K}$, i.e. much higher than $T_{T}(\sim 300 K)$. Extending the analogy with molecular diffusion (thermal diffusion), it is possible to represent the erythrocyte diffusion flux as

$$
J=-D\left(\Phi, T_{F}\right) \nabla \Phi-\zeta \nabla T_{F},
$$

where $\zeta>0$. Because collisions between the particles moving in a tube are more frequent at the periphery of the flow than near its axis, it can be expected that $T_{F}$ is non-uniformly distributed across the flow showing a maximum close to the walls. For this reason, the above equation can describe, in principle, erythrocyte depletion of the near-wall layer. Unfortunately, further development of this approach resulted in its complication; namely, the equation (4.13) was extended to include the terms corresponding to erythrocyte aggregation $\left(-D^{(N)} \nabla N\right.$, where $N$ is concentration of aggregates) and the lift forces acting in a diluted suspension [131]. In the end, the model became extremely complicated and contained a large number of unknown parameters; therefore, this work remains unfinished. However, the concept of fluctuation temperature was revived later in the simulation of dense suspensions of rigid particles (see Section 4.2.2).

4.1.5. Multiphase continuous approach Methods of this class are based on the solution of the Navier-Stokes equations written for each phase ( $k=$ plasma, erythrocytes $)$ and coupled to each other via an exchange terms:

$$
\begin{aligned}
& \sum_{k} \Phi_{k}=1 \\
& \frac{\partial\left(\rho_{k} \Phi_{k}\right)}{\partial t}+\nabla \cdot\left(\rho_{k} \Phi_{k} \bar{u}_{k}\right)=0 \\
& \frac{\partial\left(\rho_{k} \Phi_{k} \bar{u}_{k}\right)}{\partial t}+\nabla \cdot\left(\rho_{k} \Phi_{k} \bar{u}_{k} \otimes \bar{u}_{k}\right)=-\Phi_{k} \nabla p+\nabla \Sigma_{k}+\sum_{l \neq k} \beta_{k l}\left(\bar{u}_{k}-\bar{u}_{l}\right)+\bar{F}_{k}
\end{aligned}
$$

Here, $\Phi_{k}, \rho_{k}, \bar{u}_{k}$ and $\Sigma_{k}$ denote the volume fraction, density, velocity, and the shear stress tensor of phase $k$, respectively, $\beta_{k l}$ is the interphase exchange coefficient, and $\bar{F}_{k}$ stands for other forces, 
such as virtual mass and lift forces. Such representation permits to directly consider the phase relative motion, segregation, etc. The multiphase approach, unlike the single-phase one, does not require to empirically assign particle dispersion coefficients, but it implies an adequate choice of the form of tensors $\Sigma_{k}$ and the values of exchange coefficients. Such models applied to blood are used to calculate its flow in large vessels with the complicated geometry, such as aorta and coronary arteries [90-93]. Blood segregation in these vessels appears to be weak because the concentration profile has no time to develop. Accordingly, in [92, 93], the shear lift force was assumed to be close to zero, and its value in [91] was low, in agreement with weak segregation obtained. The inclusion of leukocytes as the third phase in multiphase models [93] makes it possible to describe the accumulation of these cells in some flow regions even though the validity of treating leukocytes as a continuous phase is doubtful. The two-phase micropolar model of blood was formulated in the general case in $[136,137]$. The two-phase model of suspension was extended by the addition of hydrodynamic temperature in [138].

4.2. Dense suspensions of rigid particles Consideration of the behaviour of rigid particle suspensions in a shear flow permits to disregard problems related to deformation and aggregation of the particles and focus on the role of collisions between them. The collision of two large (nonBrownian) smooth particles in a flow of viscous fluid is reversible; it means that after the collision particles return into their respective streamlines retaining the initial lateral position $[139,140]$. At the same time, this symmetry can be broken for several reasons, such as the roughness of the particles' surface, the action of interparticle repulsive forces, the influence of the third particle on the pair of colliding particles, and accumulation of genuine Brownian fluctuations in case of a sufficiently large number of collisions (the effect growing exponentially at $R e>0$ ) [27, 66, 141]. As to erythrocytes, the main cause of irreversibility of collisions between them is their deformability [134]. Thus, the collisions between the particles can by themselves contribute to their non-uniform distribution across the flow irrespective of the particle-flow interaction (see reviews in $[66,142$, 143]). The discovery of this phenomenon [140] gave a powerful impetus to rheological studies of suspensions and provided a basis for models describing the non-uniformity of particle distribution in them.

4.2.1. Phenomenological Eulerian "diffuse flux" model This approach is based on the notion of irreversibility of rebounding collisions between rough-surfaced particles [140]. It is assumed that the particles of radius $a$ moving with a carrier phase velocity along the parallel trajectories spaced less than the particle diameter undergo rebounding collisions and change lateral positions. Characteristics of this process are as follows: the displacement of each colliding particle scales as $a$, the frequency of pair collisions $\nu$ scales as $\dot{\gamma} \Phi$ (where $\Phi=V_{1} \cdot c$ is the volume fraction of the particles, $V_{1}$ is the volume of a single particle), the difference between the number of collisions in a lateral distance of $a$ scales as $\Delta \nu=a \dot{\gamma} \cdot \nabla \Phi$ [140] or $\Delta \nu=a \cdot \nabla(\dot{\gamma} \Phi)$ [144]. In the latter case, the lateral flux of particles was assessed as the sum of fluxes towards a lower collision frequency and smaller effective viscosity of the suspension: 


$$
\bar{J}=-K_{c} a^{2} \Phi \nabla(\dot{\gamma} \Phi)-K_{\eta} \frac{a^{2}}{\eta} \dot{\gamma} \Phi^{2} \nabla \eta
$$

Here, $K_{c}$ and $K_{\text {eta }}$ are the constants of order $1, \eta$ - effective viscosity of the suspension assumed to monotonically grow to infinity as the particle concentration approaches maximum package of hard spheres:

$$
\eta(\Phi)=\eta_{0} \cdot \frac{1}{\left(1-\frac{\Phi}{\Phi_{\max }}\right)^{1.82}},
$$

where $\eta_{0}$ is a medium viscosity and $\Phi_{\max }=0.68$. The steady-state concentration distribution is found by solving the equation $\mathrm{J}=0$ :

$$
\frac{\dot{\gamma} \Phi}{\dot{\gamma}_{w} \Phi_{w}}=\left(\frac{\eta\left(\Phi_{w}\right)}{\eta}\right)^{K_{\eta} / K_{c}} .
$$

This equation at $1.82\left(1-K_{\text {eta }} / K_{c}\right) \approx-1$ reduces to

$$
\Phi=\frac{\Phi_{\max }}{1+\alpha r}
$$

where $\alpha=\Phi_{\max } / \Phi_{w}-1$. This model adequately describes the experimental profile of particle concentration and the rate of its establishment in the Couette flow [144]. However, it predicts a sharp concentration peak with $\Phi=\Phi_{\max }$ at the flow axis in a tube and a concave shape of the concentration profile, in conflict with the experimental findings [142, 143, 145]. This theory was applied to the calculation of the suspension flux in a straight and a stenotic tube [146], with the particle flux given in the form equivalent to (4.15):

$$
\begin{aligned}
& J=-D_{c} \nabla \Phi-D_{s} \nabla \dot{\gamma} \\
& D_{c}=a^{2} \dot{\gamma}\left(K_{c} \Phi+K_{\eta} \Phi^{2} \frac{1}{\eta} \frac{d \eta}{d \Phi}\right) \\
& D_{s}=K_{c} a^{2} \Phi^{2}
\end{aligned}
$$

The results show the migration of particles towards the tube axis with a sharp peak of $\Phi$ on it, as in [144], and the flattened velocity profile. Thus, the "diffusive flux" model using the simplest, rough notions of collisions between particles correctly describes both the direction and the rate of their redistribution, but erroneously predicts the shape of the concentration profile.

4.2.2. Particle pressure approach Subsequent to the phenomenological "diffusive flux" model [144], a strict rheological "suspension balance" model was proposed [66, 67, 85]. In this model, the particle motion is caused by the shear stress gradient of the particle phase $\Sigma_{p}$. There are two main versions of this approach differing in the mode of taking account of the non-local dependence of this stress on the particle velocity and concentration in the suspension. In the first version, the particles are assumed to move by virtue of a gradient of positively defined particle pressure $\Pi=-\frac{1}{3} \operatorname{Tr}\left(\Sigma_{p}\right)$ or isotropic normal stress: 


$$
\begin{aligned}
& \bar{J} \sim \nabla \Pi \\
& \Pi \sim \Pi_{0}+\eta_{0} \hat{p}(\Phi) \dot{\gamma}
\end{aligned}
$$

where $\Pi_{0}$ is constant, $\hat{p}(\Phi)$ is the dimensionless monotonically growing function of the volume fraction. Evidently, the requirement of constant $\Pi$ across the flow $\left(\frac{d \Pi}{d y}=0\right)$ leads to an equation analogous to (4.16). The non-locality of the dependence of $\Pi$ on $\Phi$ and $\dot{\gamma}$ is achieved by introducing a new variable, the hydrodynamic temperature $T$, which is a measure of the particles' fluctuating motion with respect to the suspension as a whole:

$$
T=\left\langle u_{p}^{\prime} \cdot u_{p}^{\prime}\right\rangle_{p},
$$

where $u_{p}^{\prime}=u_{p}-u, \mathrm{u}$ is the mean local suspension velocity, and $<\cdot>_{p}$ denotes the ensemble average over particles. The substitution of $\dot{\gamma}$ by $a^{-1} \sqrt{T}$ in (4.18) gives

$$
\Pi=\Pi_{0}+\eta_{0} a^{-1} p(\Phi) \sqrt{T},
$$

and the condition $\frac{d \Pi}{d y}=0$ leads to the equation for $\Phi$ :

$$
\frac{d}{d y}\left(p(\Phi) T^{1 / 2}\right)=0
$$

The dependence $p(\Phi)$ is chosen in the form $p(\Phi)=\Phi^{\frac{1}{2}}\left[\left(1-\frac{\Phi}{\Phi_{m}}\right)^{-2}-1\right]$. For $T$, the balance equation of averaged fluctuation energy is written that takes into account the work of body forces, stress, dissipation of mechanical energy into heat, and fluctuation energy transfer. In the one-dimensional steady-state case, after normalization of $T$ to $(a \dot{\gamma})^{2}$, this equation reduces to

$$
\mu_{p}(\Phi)\left(\frac{d u}{d y}\right)^{2}-\alpha(\Phi) T+\varepsilon^{2} \frac{d}{d y}\left[k(\Phi) \frac{d T}{d y}\right]=0
$$

where $\mu_{p}(\Phi)=\mu_{a}(\Phi)-1, \mu_{a}(\Phi)$ is the relative suspension viscosity, $\varepsilon=a / H, \alpha(\Phi)$ and $k(\Phi)$ are the phenomenological coefficients. The balance of the moment of momentum in the steady-state has the form

$$
\frac{d}{d y}\left(\mu_{a}(\Phi) \frac{d u}{d y}\right)=-1
$$

The values of $u, T$, and $\Phi$ are found by solving the equations (4.21) - (4.23) with the boundary conditions of symmetry $u$ and $T$ on the axis $(y=0)$ and $u=T=0$ on the wall $(y= \pm 1)$. For $\Phi$, the integral condition of conservation of the total number of particles is assumed. The solution of this system describes the accumulation of particles in the near-axis region with $\Phi<\Phi_{m}$ on the axis. However, it gives an erroneous maximum of $\Phi$ close to the wall eliminated by the substitution of the boundary condition for $T$ by [67]

$$
T_{(y= \pm 1)}=\frac{\mu_{p}\left(\Phi_{w}\right)}{\alpha^{*}}\left(\frac{d u}{d y}\right)_{(y= \pm 1)}^{2},
$$


where $\alpha^{*} \sim 20 \alpha\left(\Phi_{w}\right)$. The thorough comparison of particle concentration and velocity profiles predicted by this model with experimental observations and simulations of Stokesian dynamics demonstrated a fairly good agreement between them [66, 67, 143, 147].

The second version of the "suspension balance" model is related to the discovery of anisotropy of $\Sigma_{p}$ and $T$ in curvilinear and tubular flows $[85,148]$. The particle flux is assumed to be

$$
\bar{J}=\frac{2}{9} a^{2} \frac{f(\Phi)}{\eta_{0}} \nabla \Sigma_{p}
$$

where $f(\Phi)$ is the mean particle mobility. The equation for the shear stress tensor of the disperse phase $\Sigma_{p}$ is written out by analogy with that for the shear stress tensor of the carrier phase:

$$
\begin{aligned}
& \Sigma_{p}=\Sigma_{p}^{N S}+2 \eta_{0} \cdot\left(\mu_{a}-1\right) \cdot d \\
& \Sigma_{f}=-p I+2 \eta_{0} \cdot d
\end{aligned}
$$

(here, $\eta_{0}$ is a carrier phase viscosity, $\mu_{a}$ - relative shear viscosity of suspension, $p$ - pressure) so that the sum of them gives the shear stress tensor of the suspension:

$$
\Sigma=\Sigma_{p}+\Sigma_{f}=-p I+2 \eta_{0} \mu_{a} \cdot d+\Sigma_{p}^{N S} .
$$

Note that this expression is different from generalized Newton's law (4.3) in that its last item is the normal shear stress tensor of the disperse phase. In this version of the "suspension balance" model, the concept of hydrodynamic temperature is substituted by the non-local dependence $\Sigma_{p}^{N S}(\dot{\gamma})$ introduced by averaging $\dot{\gamma}$ over a certain vicinity of a given point. In the simplest case, $\dot{\gamma}$ is supplemented by a small constant $\dot{\gamma}_{N L}$ proportional to the wall shear rate. It influences the solution only on the axis, where $\dot{\gamma}=0$ :

$$
\Sigma_{p}^{N S}=-\eta_{0} \mu_{n}(\Phi)\left[\dot{\gamma}(x)+\dot{\gamma}_{N L}\right] Q .
$$

Here, $\mu_{n}$ is relative normal stress viscosity, $Q$ is the tensor parameter of anisotropy the directions of which correspond to velocity, gradient, and vortex respectively:

$$
Q=\left(\begin{array}{ccc}
1 & 0 & 0 \\
0 & \lambda_{3} & 0 \\
0 & 0 & \lambda_{3}
\end{array}\right)
$$

with $\lambda_{2} \approx 0.8, \lambda_{3} \approx 0.5$. This approach also gave a good description of the migration of particles towards the flow axis with $\Phi<\Phi_{m}$ on it. Again, the most pronounced deviations from the experimental concentration profiles occurred in the near-wall region; probably, modification of the boundary conditions is needed.

4.3. Non-uniform platelet distribution in blood In the overwhelming majority of the works where the platelet concentration is assumed to be a continuous coordinate function $P(\bar{x})$ [149-160], it is found by solving the convection-diffusion equation of the form (4.8), where $P$ is substituted for $\Phi$ and the flux $\bar{J}$ is given according to Fick's law: 


$$
\bar{J}=-D \nabla P .
$$

Here, $D$ is the effective diffusion coefficient. Evidently, the non-uniform platelet distribution across the flow cannot be described in this way. The phenomenological description of the nonuniform platelet concentration profile in the presence of erythrocytes was obtained only after the introduction of a potential term into this equation (drift-flux model) [161, 162]:

$$
\bar{J}=-D \nabla P-P \nabla \Psi .
$$

Here, the entire lateral platelet flux equals to the sum of random and directed motions. The "rheological potential" $\Psi$ reflects the overall directional effect of erythrocytes on platelets in a shear flow: the velocity of their lateral motion ("drift shape function") equals to $V_{\text {drift }}=-\nabla \Psi$. In a stationary parallel flow, this function was determined by differentiation of the concentration profiles $P_{0}(y)$ of platelet-sized latex spheres in blood [163]. At $\bar{J}=0$ one has

$$
\frac{V_{d r i f t}(y)}{D}=\frac{1}{P_{0}} \frac{d P_{0}}{d y} .
$$

In a flow with the non-parallel geometry, the lateral velocity was assumed to be augmented in a shear rate-dependent manner [105]

$$
V_{d r i f t}(r)=\frac{D}{P_{0}} \frac{d P_{0}}{d y}+a_{P} \dot{\gamma}
$$

where $a_{P}$ is the platelet size. The necessity of experimental determination of the drift shape function is a serious drawback of the present method that precludes a-priori prediction of platelet distribution.

\section{General view of equations of continuum models of blood flow: Summary}

The above models can be generalized as follows. The equation of suspension motion as a whole (continuity and conservation of momentum) has the form

$$
\begin{aligned}
& \nabla \cdot \bar{u}=0 \\
& \frac{D \bar{u}}{D t}=\frac{1}{\rho} \nabla \Sigma
\end{aligned}
$$

When blood is considered as a generalized Newtonian medium, the rheological equation has the form (4.3):

$$
\Sigma=-p I+2 \eta_{0} \mu_{a} \cdot d
$$

Here, the relative dynamic viscosity $\mu_{a}$ is given by one of the models presented in Section 4.1.1 (the Carreau and Quemada models are preferred). The equations of motion for suspended particles (4.8) 


$$
\frac{D \Phi}{D t}=-\nabla \bar{J}
$$

which is necessary to obtain their non-uniform distribution, requires prescription of an expression for $\bar{J}$. Because no well-founded form of this expression has thus far been proposed, we shall use analogies with the known models. To begin with, a lateral flux of erythrocytes may be a consequence of balance between their lateral migration resulting from interactions of individual cells with the carrier phase (blood plasma) and collisional shear-induced diffusion leveling their concentration:

$$
\bar{J}=\Phi \cdot \bar{V}_{l a t}-D \nabla \Phi .
$$

The expression for the diffusion coefficient may be an approximation of the experimental data [164] or calculated, for instance, by formula (4.17). As follows from the Section 2.2, the expression for $\bar{V}_{\text {lat }}$ should resemble the formula for lateral velocity of particles undergoing deformation in a diluted suspension (2.8-2.10), probably with the correction for the local erythrocyte concentration.

Another possibility to obtain the expression for $\bar{J}$ arises from the assumption of collisions between erythrocytes as the main source of their lateral migration. In this case, (see Section 4.2.2), the analog of the expression for the flux of particles in concentrated suspensions can be written in the form

$$
\begin{gathered}
\bar{J}=\frac{2}{9} a^{2} \frac{f(\Phi)}{\eta_{0}} \nabla \Sigma_{p} \\
\Sigma_{p}=\Sigma_{p}^{N S}+2 \eta_{0} \cdot\left(\mu_{a}-1\right) \cdot d .
\end{gathered}
$$

The simplest form of expression for the pressure of erythrocytes is

$$
\Sigma_{p}^{N S}=-\eta_{0} \cdot \alpha(\Phi) \cdot \dot{\gamma} \cdot I
$$

Here, the tensor $Q$ is simplified to the unit tensor $I$ and $\alpha(\Phi)$ is the monotonically growing function of hematocrit. The right hand side of this expression is equal to $-\Pi \cdot I$, where $\Pi=\eta_{0} \cdot \alpha(\Phi) \cdot \dot{\gamma}$. In this case, (5.2) is replaced by

$$
\Sigma=-p I+2 \eta_{0} \mu_{a} \cdot d+\Sigma_{p}^{N S} .
$$

The non-local character of the erythrocyte-erythrocyte interaction should be taken into consideration by local (over a region measuring roughly several erythrocyte dimensions) averaging of $\dot{\gamma}$ in (5.7) in accordance with (4.27). However, a more general approach is to write out the equation for suspension temperature $T$. Generalizing (4.22) and substituting $\dot{\gamma}$ by $a^{-1} \sqrt{T}$ in (5.7) yield

$$
\begin{gathered}
\mu_{p}(\Phi) \dot{\gamma}^{2}-\alpha(\Phi) T+\varepsilon^{2} \nabla \cdot[k(\Phi) \nabla T]=0 \\
\Sigma_{p}^{N S}=-\eta_{0} \cdot \alpha(\Phi) \cdot a^{-1} \sqrt{T} \cdot I
\end{gathered}
$$


It is still uncertain which of the above approaches matches the real situation in blood. Further studies must be designed to clarify this uncertainty.

\section{Well-posedness of flow models}

Different models discussed in the present paper are given by a system of partial differential equations (PDEs) equipped with some boundary conditions and (in time-dependent models) with some initial conditions. These models may be expressed by the equations

$$
L u=f,
$$

where the right hand side $f$ stands for the set of given right hand sides for the PDE, boundary and initial conditions, $u$ stands for the vector of unknown functions (for example, the velocity, the pressure, the concentrations), and $L$ is an operator (often non-linear), relating the data $f$ and the unknowns $u$ in some appropriate spaces.

The rigorous mathematical approach considers the well-posedness of such models as a "trinity" of properties: existence and uniqueness of the solution of equation (6.1) and stability of this equation. Normally, $L$ is defined as an operator $L: B_{1} \rightarrow B_{2}$, where $B_{1}$ and $B_{2}$ are some Euclidean spaces equipped with the norms $\|\cdot\|_{1}$ and $\|\cdot\|_{2}$, respectively. The stability of (6.1) means that for any couple of data $f_{1}$ and $f_{2}$ from $B_{2}$ (from some ball in $B_{2}$ ) the solutions $u_{1}$ and $u_{2}$ of problems $L u_{1}=f_{1}$ and $L u_{2}=f_{2}$ satisfy the estimate

$$
\left\|u_{1}-u_{2}\right\|_{1} \leq C\left\|f_{1}-f_{2}\right\|_{2},
$$

where $C$ is a constant independent of $f_{1}$ and $f_{2}$. This estimate is an important tool for the justification of approximate methods for solution of equation (6.1): assume that we constructed an approximate solution $u_{a}$ of (6.1) satisfying it with some discrepancy $r$, i.e.

$$
L u_{a}=f+r,
$$

where $r$ has a small norm in $B_{2}$. Then from (6.1)-(6.3) we get an estimate for $u_{a}$ :

$$
\left\|u-u_{a}\right\|_{1} \leq C\|r\|_{2} .
$$

Unfortunately this well-posedness is not proved for a great number of models (mainly non-linear) important for applications. So normally, these problems are solved by approximate methods that are justified for some "model problems" that are often some linearized analogues of the real problems. As a classical example we could give the linear Stokes equations or the Ozeen equations that "imitate" essentially more complicated the Navier-Stokes equations (4.2)-(4.5). Of course, such "imitation" may be very far from the reality. Some results on the existence and uniqueness of solutions one can find in [165-167]. 


\section{Conclusion}

Generally speaking, the Lagrangian methods especially LBM are currently preferred for the description of the main effects of erythrocyte and platelet segregation in diluted suspensions and blood flowing in microvessels. The quantitative agreement with the experimental observations is achieved at both micro- (non-uniform distribution of concentrations) and macro- (Fåhræus and Fåhræus-Lindqvist effects) levels. Parallelization of LBM and DEM is relatively easy to perform and the computational cost linearly depends on the number of particles, in contrast to PDE-based methods which are difficult to parallelize and have a quadratic dependence of computational cost on the mesh density. These properties of LBM and DEM make them the promising tools for the solution of blood dynamics problems. However, their drawbacks, such as the necessity of large amounts of computation, the stochastic nature of results, and the difficulty of analytical treatment, strongly require the development of efficient continuous methods.

Traditional rheological models of blood and the majority of Eulerian models derived from them to simulate bloodflow disregard the non-uniform lateral distribution of erythrocytes. Therefore, they are applicable only under conditions of weak blood segregation - uniform shear flow in vitro and macrocirculatory vessels. Blood segregation could be described in terms of the multiphase models, but their application for this purpose have up to now been confined to the cases of very weak segregation. The micropolar models provide a fairly good description of blood segregation, but their physical relevance to the blood flow conditions is questionable because of the highly chaotic motion and deformation of erythrocytes due to their continuous collisions with each other and because of weak influence of particle rotation on their lateral migration in a viscous flow.

In this review, we proposed two alternative continuous approaches to the description of lateral migration of erythrocytes based on two different assumptions about the ruling mechanism of this process. The first approach assumes that the migration of erythrocytes is similar to that of suspended droplets and vesicles, i.e. migration results from their interaction with the surrounding fluid and deformation. It leads to the equation (5.4) which describes the balance between the directed migration of individual erythrocytes and their shear-induced dispersion resulting from collisions. The second approach uses the analogy with the migration of suspended rigid particles and assumes that the erythrocyte-erythrocyte interactions in the shear flow drive their lateral migration. It leads to the equations (5.5)-(5.7) wich connect the erythrocyte flux and the shear stress tensor of the disperse phase; on accounting for the non-locality of interactions (using the suspension temperature), equation (5.7) substitutes for (5.9)-(5.10). At present, the choice between these two approaches is a challenging problem. The numerous data suggesting the central role of erythrocyte deformability in their lateral migration and augmentation of migration with increasing deformation argue in favour of the former approach. In contrast, deformability of erythrocytes must hamper their lateral migration by the mechanism assumed in the second approach because the fluctuation energy is spent to deform the cell membrane and contents. At the same time, erythrocytes exhibit certain properties of rigid particles; for this reason the second approach can not be altogether neglected either. Possibly, the two approaches either separately or in combination should be taken into consideration in the quantitative description of blood segregation. 


\section{Acknowledgements}

This work is a part of joint French-Russian Programme International de Cooperation Scientifique (PICS) "Mathematical modelling of blood diseases". It was supported in part by grants 09-0492427, 09-02-00018, 09-04-00232, 09-04-00357, 10-01-91055 and 10-04-00035 from the Russian Foundation for Basic Research.

\section{References}

[1] C. G. Caro, T. J. Pedley, R. C. Schroter, W. A. Seed. The mechanics of the circulation (russian edition). Mir, Moscow, 1981.

[2] A. M. Chernukh, P. N. Aleksandrov, O. V. Alekseev. Microcirculation. Medicina, Moscow, 1984.

[3] H. L. Goldsmith, R. Skalak. Hemodynamics. Annual Review of Fluid Mechanics, 7 (1975), 213-247.

[4] H. L. Goldsmith, V. T. Turitto. Rheological aspects of thrombosis and haemostasis: basic principles and applications. ICTH-Report-Subcommittee on Rheology of the International Committee on Thrombosis and Haemostasis. Thromb. Haemost., 55 (1986), No. 3, 415-435.

[5] H. L. Goldsmith. The Microcirculatory Society Eugene M. Landis Award lecture. The microrheology of human blood. Microvasc. Res., 31 (1986), No. 2, 121-142.

[6] A. S. Popel, P. C. Johnson. Microcirculation and hemorheology. Annu. Rev. Fluid Mech., 37 (2005), 43-69.

[7] H. H. Lipowsky. Microvascular rheology and hemodynamics. Microcirculation, 12 (2005), 5-15.

[8] G. R. Cokelet. Viscometric, in vitro and in vivo blood viscosity relationships: how are they related? (Poiseuille Award Lecture). Biorheology, 36 (1999), 343-358.

[9] A. M. Quarteroni, M. Tuveri, A. Veneziani. Computational vascular fluid dynamics: problems, models and methods. Computing and Visualization in Science, 2 (2000), 163-197.

[10] S. Kim, P. K. Ong, O. Yalcin, M. Intaglietta, P. C. Johnson. The cell-free layer in microvascular blood flow. Biorheology, 46 (2009), 181-189.

[11] M. Manjunatha, M. Singh. Digital blood flow analysis from microscopic images of mesenteric microvessel with multiple branching. Clin. Hemorheol. Microcirc., 27 (2002), 91-106.

[12] M. Manjunatha, S. S. Singh, M. Singh. Blood flow analysis in mesenteric microvascular network by image velocimetry and axial tomography. Microvascular Research, 65 (2003), 49-55. 
[13] A. A. Palmer, W. H. Betts. The axial drift of fresh and acetaldehyde-hardened erythrocytes in 25 mum capillary slits of various lengths. Biorheology, 12 (1975), No. 5, 283-293.

[14] M. L. Ellsworth, R. N. Pittman. Evaluation of photometric methods for quantifying convective mass transport in microvessels. Am. J. Physiol., 251 (1986), H869-H879.

[15] A. R. Pries, K. Ley, M. Claassen, P. Gaehtgens. Red Cell Distribution at Microvascular Bifurcations. Microvasc. Res., 38 (1989), 81-101.

[16] R. H. Phibbs. Distribution of leukocytes in blood flowing through arteries. Am. J. Physiol., 210 (1966), No. 5, 919-925.

[17] G. J. Tangelder, H. C. Teirlinck, D. W. Slaaf, R. S. Reneman. Distribution of blood platelets flowing in arterioles. Am. J. Physiol., 248 (1985), H318-H323.

[18] B. Woldhuis, G. J. Tangelder, D. W. Slaaf, R. S. Reneman. Concentration profile of blood platelets differs in arterioles and venules. Am. J. Physiol., 262 (1992), H1217-H1223.

[19] P. A. Aarts, S. A. van den Broek, G. W. Prins, G. D. Kuiken, J. J. Sixma, R. M. Heethaar. Blood platelets are concentrated near the wall and red blood cells, in the center in flowing blood. Arteriosclerosis, 8 (1988), No. 6, 819-824.

[20] H. L. Goldsmith. Red cell motions and wall interactions in tube flow. Fed. Proc., 30 (1971), No. 5, 1578-1590.

[21] G. Segré, A. Silberberg. Radial particle displacements in poiseuille flow of suspensions. Nature, 189 (1961), 209-210.

[22] G. Segré, A. Silberberg. Behaviour of macroscopic rigid spheres in Poiseuille flow Part 1. Determination of local concentration by statistical analysis of particle passages through crossed light beams. Journal of Fluid Mechanics, 14 (1962), 115-135.

[23] G. Segré, A. Silberberg. Behaviour of macroscopic rigid spheres in Poiseuille flow Part 2. Experimental results and interpretation. Journal of Fluid Mechanics, 14 (1962), 136-157.

[24] D. R. Oliver. Influence of particle rotation on radial migration in the Poiseuille flow of suspensions. Nature, 194 (1962), 1269-1271.

[25] M. Takano, H. L. Goldsmith, S. G. Mason. The flow of suspensions through tubes VIII. Radial Migration of Particles in Pulsatile Flow. Journal of Colloid and lnterface Science, 27 (1968), No. 2, 253-267.

[26] L. G. Leal. Particle motions in a viscous fluid. Annu. Rev. Fluid Mech., 12 (1980), 435-476.

[27] S. K. Wang, N. H. C. Hwang. On transport of suspended particulates in tube flow. Biorheology, 29 (1992), 353-377. 
[28] H. Brenner, P. M. Bungay. Rigid-particle and liquid-droplet models of red cell motion in capillary tubes. Fed. Proc., 30 (1971), No. 5, 1565-1577.

[29] H. L. Goldsmith, S. G. Mason. The flow of suspensions through tubes I. Single spheres, rods and discs. Journal of Colloid Science, 17 (1962), 448-476.

[30] C. K. W. Tam, W. A. Hyman. Transverse motion of an elastic sphere in a shear field. Journal of Fluid Mechanics, 59 (1973), No. part 1, 177-185.

[31] C. Crowe, M. Sommerfield, Y. Tsuji. Multiphase flows with drops and particles. CRC Press, 1998.

[32] P. Cherukat, J. B. McLaughlin, D. S. Dandy. A computational study of the inertial lift on a sphere in a linear shear fow field. International Journal of Multiphase Flow, 25 (1999), 15-33.

[33] J.-P. Matas, J. F. Morris, E. Guazzelli. Inertial migration of rigid spherical particles in Poiseuille flow. Journal of Fluid Mechanics, 515 (2004), 171-195.

[34] L. L. Munn, M. M. Dupin. Blood cell interactions and segregation in flow. Ann. Biomed. Eng, 36 (2008), No. 4, 534-544.

[35] E. E. Michaelides. Hydrodynamic Force and Heat-Mass Transfer From Particles. Journal of Fluids Engineering, 125 (2003), 209-238.

[36] S. I. Rubinow, J. B. Keller. The transverse force on a spinning sphere moving in a viscous fluid. Journal of Fluid Mechanics, 11 (1961), 447-459.

[37] R. G. Cox, H. Brenner. The lateral migration of solid particles in Poiseuille flow - I theory. Chemical Engineering Science, 23 (1968), 147-173.

[38] P. G. Saffman. The lift on a small sphere in a slow shear flow. Journal of Fluid Mechanics, 22 (1965), No. part 2, 385-400.

[39] J. B. McLaughlin. Inertial migration of a small sphere in linear shear flows. Journal of Fluid Mechanics, 224 (1991), 261-274.

[40] P. Cherukat, J. B. McLaughlin, A. L. Graham. The inertial lift on a rigid sphere translating in a linear shear flow field. International Journal of Multiphase Flow, 20 (1994), No. 2, 339-353.

[41] P. V. Vasseur, R. G. Cox. The lateral migration of a spherical particle in two-dimensional shear flows . Journal of Fluid Mechanics, 78 (1976), 385-413.

[42] R. G. Cox, S. K. Hsu. The lateral migration of solid particles in a laminar flow near a plane. International Journal of Multiphase Flow, 3 (1977), 201-222.

[43] P. Cherukat, D. R. Oliver. The inertial lift on a rigid sphere in a linear shear flow field near a flat wall. Journal of Fluid Mechanics, 263 (1994), 1-18. 
[44] P. W. Longest, C. Kleinstreuer. Comparison of blood particle deposition models for nonparallel fow domains. Journal of Biomechanics, 36 (2003), 421-430.

[45] P. W. Longest, C. Kleinstreuer. Numerical Simulation of Wall Shear Stress Conditions and Platelet Localization in Realistic End-to-Side Arterial Anastomoses. Journal of Biomechanical Engineering, 125 (2003), 671-681.

[46] P. W. Longest, C. Kleinstreuer, J. R. Buchanan. Efficient computation of micro-particle dynamics including wall effects. Computers \& Fluids, 33 (2004), 577-601.

[47] H. L. Goldsmith, S. G. Mason. Axial migration of particles in Poiseuille Flow. Nature, 190 (1961), 1095-1096.

[48] M. Abkarian, A. Viallat. Vesicles and red blood cells in shear flow. Soft Matter, 4 (2008), 653-657.

[49] C. Coulliette, C. Pozrikidis. Motion of an array of drops through a cylindrical tube. Journal of Fluid Mechanics, 358 (1998), 1-28.

[50] S. Mortazavi, G. Tryggvason. A numerical study of the motion of drops in Poiseuille flow. Part 1.Lateral migration of one drop. Journal of Fluid Mechanics, 411 (2000), 325-350.

[51] C. Pozrikidis. Numerical Simulation of Cell Motion in Tube Flow. Ann. Biomed. Eng, 33 (2005), No. 2, 165-178.

[52] B. Kaoui, G. Biros, C. Masbah. Why Do Red Blood Cells Have Asymmetric Shapes Even in a Symmetric Flow? Physical Review Letters, 103 (2009), No. 18, 188101(1)-188101(4).

[53] C. E. Chaffey, H. Brenner, S. G. Mason. Particle motions in sheared suspensions XVIII. Wall Migration (Theoretical). Rheologica Acta, 4 (1965), No. 1, 64-72.

[54] C. E. Chaffey, H. Brenner, S. G. Mason. Correction of the paper Particle motions in sheared suspensions XVIII. Wall Migration (Theoretical). Rheologica Acta, 6 (1967), No. 1, 100.

[55] P. R. Wohl, S. I. Rubinow. The transverse force on a drop in an unbounded parabolic flow. Journal of Fluid Mechanics, 62 (1974), No. part 1, 185-207.

[56] P. C. H. Chan, L. G. Leal. The motion of a deformable drop in a second-order fluid. Journal of Fluid Mechanics, 92 (1979), No. part 1, 131-170.

[57] W. S. J. Uijttewaal, E.-J. Nijhof, R. M. Heethaar. Droplet migration, deformation, and orientation in the presence of a plane wall: A numerical study compared with analytical theories. Phys. Fluids A, 5 (1993), No. 4, 819-825.

[58] S. D. Hudson. Wall migration and shear-induced diffusion of fluid droplets in emulsions. Physics of Fluids, 15 (2003), No. 5, 1106-1113. 
[59] M. R. King, D. T. Leighton, Jr. Measurement of shear-induced dispersion in a dilute emulsion. Physics of Fluids, 13 (2001), No. 2, 397-406.

[60] M. Scott. 2005. The modeling of Blood Rheology in small vessels. University of Waterloo, Waterloo, Ontario, Canada.

[61] P. Olla. The lift on a tank-treading ellipsoidal cell in a shear flow. Journal de Physique II, 7 (1997), No. 10, 1533-1540.

[62] M. Faivre, M. Abkarian, K. Bickraj, H. A. Stone. Geometrical focusing of cells in a microfluidic device: An approach to separate blood plasma. Biorheology, 43 (2006), 147-159.

[63] P. L. Blackshear, Jr., R. J. Forstrom, F. D. Dorman, G. O. Voss. Effect of flow on cells near walls. Fed. Proc., 30 (1971), No. 5, 1600-1609.

[64] C. D. Eggleton, A. S. Popel. Large deformation of red blood cell ghosts in a simple shear flow. Physics of Fluids, 10 (1998), No. 8, 1834-1845.

[65] N. Korin, A. Bransky, U. Dinnar. Theoretical model and experimental study of red blood cell (RBC) deformation in microchannels. Journal of Biomechanics, 40 (2007), 2088-2095.

[66] P. R. Nott, J. F. Brady. Pressure-driven flow of suspensions: simulation and theory. Journal of Fluid Mechanics, 275 (1994), 157-199.

[67] J. F. Morris, J. F. Brady. Pressure-driven flow in a suspension: buoyancy effects. International Journal of Multiphase Flow, 24 (1998), No. 1, 105-130.

[68] K. Tsubota, S. Wada, H. Kamada, Y. Kitagawa, R. Lima, T. Yamaguchi. A Particle Method for Blood Flow Simulation -Application to Flowing Red Blood Cells and Platelets. Journal of the Earth Simulator, 5 (2006), 2-7.

[69] S. Chen, G. D. Doolen. Lattice Boltzmann Method for Fluid Flows. Annu. Rev. Fluid Mech., 30 (1998), 329-364.

[70] M. M. Dupin, I. Halliday, C. M. Care, L. Alboul, L. L. Munn. Modeling the flow of dense suspensions of deformable particles in three dimensions. Physical Review E., 066707 (2007), $1-17$.

[71] L. M. Crowl, A. L. Fogelson. Computational model of whole blood exhibiting lateral platelet motion induced by red blood cells. Commun. Numer. Meth. Engng, (2009).

[72] C. Sun, C. Migliorini, L. L. Munn. Red Blood Cells Initiate Leukocyte Rolling in Postcapillary Expansions: A Lattice Boltzmann Analysis. Biophysical Journal, 85 (2003), 208-222.

[73] P. Bagchi. Mesoscale Simulation of Blood Flow in Small Vessels. Biophysical Journal, 92 (2007), 1858-1877. 
[74] P. Bagchi, P. C. Johnson, A. S. Popel. Computational Fluid Dynamic Simulation of Aggregation of Deformable Cells in a Shear Flow. Transactions of the ASME, 127 (2005), 1070-1080.

[75] J. Zhang, P. C. Johnson, A. S. Popel. Red blood cell aggregation and dissociation in shear flows simulated by lattice Boltzmann method. Journal of Biomechanics, 41 (2008), 47-55.

[76] J. Zhang, P. C. Johnson, A. S. Popel. Effects of erythrocyte deformability and aggregation on the cell free layer and apparent viscosity of microscopic blood flows. Microvasc. Res., 77 (2009), 265-272.

[77] S. Svetina, P. Ziherl. Morphology of small aggregates of red blood cells. Bioelectrochemistry, 73 (2008), No. 2, 84-91.

[78] A. L. Fogelson. A Mathematical Model and Numerical Method for Studying Platelet Adhesion and Aggregation during Blood Clotting. Journal of Computational Physics, 56 (1984), 111-134.

[79] I. V. Pivkin, P. D. Richardson, G. Karniadakis. Blood flow velocity effects and role of activation delay time on growth and form of platelet thrombi. PNAS, 103 (2006), No. 46, $17164-$ 17169.

[80] H. Miyazaki, T. Yamaguchi. Formation and destruction of primary thrombi under the influence of blood flow and von Willebrand factor analyzed by a discrete element method. Biorheology, 40 (2003), 265-272.

[81] K. Yano, K. Tsubota, S. Wada, T. Yamaguchi. 2003. Computational mechanical simulation of the aggregation and adhesion of platelets in the blood flow. In Summer Bioengineering Conference. Sonesta Beach Resort in Key Biscayne, Florida. 0613-0614.

[82] N. Filipovic, D. Ravnic, M. Kojic, S. J. Mentzer, S. Haber, A. Tsuda. Interactions of blood cell constituents: Experimental investigation and computational modeling by discrete particle dynamics algorithm. Microvasc. Res., 75 (2008), 279-284.

[83] D. Mori, K. Yano, K. Tsubota, T. Ishikawa, S. Wada, T. Yamaguchi. Simulation of platelet adhesion and aggregation regulated by fibrinogen and von Willebrand factor. Thromb. Haemost., 99 (2008), No. 1, 108-115.

[84] T. Almomani, H. S. Udaykumar, J. S. Marshall, K. B. Chandran. Micro-scale dynamic simulation of erythrocyte-platelet interaction in blood flow. Ann. Biomed. Eng, 36 (2008), No. 6, 905-920.

[85] R. M. Miller, J. F. Morris. Normal stress-driven migration and axial development in pressuredriven flow of concentrated suspensions. Journal of Non-Newtonian Fluid Mechanics, 135 (2006), 149-165.

[86] L. G. Loitzanskii. Mechanics of Fluid and Gas. Nauka, Moscow, 1978. 
[87] A. Sequeira, J. Janela. An overview of some mathematical models of blood rheology. In A Portrait of State-of-the-Art Research at the Technical University of Lisbon. M. S. Pereira, editor. Springer, 2007. pp. 65-87.

[88] A. M. Robertson, A. Sequeira, M. V. Kameneva. Hemorheology. In Hemodynamical Flows: Modeling, Analysis and Simulation (Oberwolfach Seminars). Birkhauser Basel, 2008. pp. 63-120.

[89] G. R. Cokelet. The Rheology and Tube Flow of Blood. In Handbook of Bioengineering. R. Skalak, S. Chen, editors. McGraw-Hill, New York, 1987.

[90] B. J. B. M. Wolters, M. C. M. Rutten, G. W. H. Schurink, U. Kose, J. d. Hart, F. N. v. d. Vosse. A patient-specific computational model of fluid-structure interaction in abdominal aortic aneurysms. Medical Engineering \& Physics, 27 (2005), 871-883.

[91] J. Jung, A. Hassenein, R. W. Lyczkowski. Hemodynamic Computation Using Multiphase Flow Dynamics in a Right Coronary Artery. Ann. Biomed. Eng, 34 (2006), No. 3, 393-407.

[92] J. Jung, R. W. Lyczkowski, C. B. Panchal, A. Hassenein. Multiphase hemodynamic simulation of pulsatile flow in a coronary artery. Journal of Biomechanics, 39 (2006), 2064-2073.

[93] J. Jung, A. Hassenein. Three-phase CFD analytical modeling of blood flow. Medical Engineering \& Physics, 30 (2008), 91-103.

[94] D. Quemada, C. Berli. Energy of interaction in colloids and its implications in rheological modeling. Advances in Colloid and Interface Science, 98 (2002), 51-85.

[95] A. Marcinkowska-Gapinska, J. Gapinski, W. Elikowski, F. Jaroszyk, L. Kubisz. Comparison of three rheological models of shear flow behavior studied on blood samples from postinfarction patients. Medical and Biological Engineering and Computing, 45 (2007), No. 9 , 837-844.

[96] B. Das, P. C. Johnson, A. S. Popel. Effect of nonaxisimmetric hematoctit distribution on nonnewtonian blood flow in small tubes. Biorheology, 35 (1998), No. 1, 69-87.

[97] J. R. Buchanan, Jr., C. Kleinstreuer, J. K. Comer. Rheological effects on pulsatile hemodynamics in a stenosed tube. Computers \& Fluids, 29 (2000), 695-724.

[98] B. Das, G. Enden, A. S. Popel. Stratified multiphase model for blood flow in a venular bifurcation. Annals of Biomedical Engineering, 25 (1997), 135-153.

[99] A. S. Popel, G. Enden. An analytical solution for steady flow of a Quemada fluid in a circular tube. Rheologica Acta, 32 (1993), 422-426.

[100] C. L. Berli, D. Quemada. Aggregation behavior of red blood cells in shear flow. A theoretical interpretation of simultaneous rheo-optical and viscometric measurements. Biorheology, 38 (2001), No. 1, 27-38. 
[101] D. Quemada. Rheological modelling of complex fluids. I. The concept of effective volume fraction revisited. The European Physical J. AP, 1 (1998), 119-127.

[102] D. Quemada, C. Berli. Energy of interaction in colloids and its implications in rheological modeling. Adv. Colloid Interface Sci., 98 (2002), No. 1, 51-85.

[103] P. Neofytou. Comparison of blood rheological models for physiological flow simulation. Biorheology, 41 (2004), No. 6, 693-714.

[104] G. R. Cokelet, H. L. Goldsmith. Decreased hydrodynamic resistance in the two-phase flow of blood through small vertical tubes at low flow rates. Circ. Res., 68 (1991), No. 1, 1-17.

[105] J. R. Buchanan, Jr., C. Kleinstreuer. Simulation of particle-hemodynamics in a partially occluded artery segment with implications to the initiation of microemboli and secondary stenoses. J. Biomech. Eng, 120 (1998), No. 4, 446-454.

[106] S. A. Regirer. Lections on Biological Mechanics [in russian]. Izdatelstvo MGU, Moscow, 1980.

[107] M. Sharan, A. S. Popel. A two-phase model for flow of blood in narrow tubes with increased effective viscosity near the wall. Biorheology, 38 (2001), 415-428.

[108] J. H. Ware, F. Y. Sorrell, R. M. Felder. A model of steady blood flow. Biorheology, 11 (1974), 97-109.

[109] B. Das, P. C. Johnson, A. S. Popel. Computational fluid dynamic studies of leukocyte adhesion effects. Biorheology, 37 (2000), 239-258.

[110] J. Perkkio, R. Keskinen. On the effect of the concentration profile of red cells on blood flow in the artery with stenosis. Bull. Math. Biol., 45 (1983), No. 2, 259-267.

[111] D. Lerche. Modelling hemodynamics in small tubes (hollow fibers) considering . In Biomechanical Transport Processes. F. e. al. Mosora, editor. Plenum, New York, 1990. pp. 243-250.

[112] R. T. Carr, M. Lacoin. Nonlinear Dynamics of Microvascular Blood Flow. Annals of Biomedical Engineering, 28 (2000), 641-652.

[113] P. Brunn. The general solution to the equations of creeping motion of a micropolar fluid and its application. International Journal of Engineering Science, 20 (1982), 575-585.

[114] V. K. Stokes. Couple stress in fluids. The Physics of Fluids, 9 (1966), No. 9, 1709-1715.

[115] A. C. Eringen. Theory of Micropolar Fluids. Journal of Mathematics and Mechanics, 16 (1966), No. 1, 1-18.

[116] A. Askar, A. S. Cakmak. A structural model of a micropolar continuum. International Journal of Engineering Science, 6 (1968), 583-589. 
[117] T. Ariman. Microcontinuum fluid mechanics - a review. International Journal of Engineering Science, 11 (1973), 905-930.

[118] T. Ariman, M. A. Turk, N. D. Sylvester. Application of microcontinuum fluid mechanics. International Journal of Engineering Science, 12 (1974), 273-293.

[119] K. A. Kline. Predictions from Polar Fluid Theory Which Are Independent of Spin Boundary Condition. Transactions of the society of rheology, 19 (1975), No. 1, 139-145.

[120] S. C. Cowin. A Note on the Predictions from Polar Fluid Theory Which Are Independent of the Spin Boundary Condition. Transactions of the society of rheology, 20 (1976), No. 2, 195-202.

[121] H. A. Hogan, M. Henriksen. An evaluation of a micropolar model for blood flow through an idealized stenosis. Journal of Biomechanics, 22 (1989), No. 3, 211-218.

[122] R. N. Pralhad, D. H. Schultz. Modeling of arterial stenosis and its applications to blood diseases. Mathematical Biosciences, 190 (2004), 203-220.

[123] G. Akay, A. Kaye. Numerical solution of time dependent stratified two-phase flow of micropolar fluids and its application to flow of blood through fine capillaries. International Journal of Engineering Science, 23 (1985), No. 3, 265-276.

[124] Md. A. Ikbal, S. Chakravarty, P. K. Mandal. Two-layered micropolar fluid flow through stenosed artery: Effect of peripheral layer thickness. Computers and Mathematics with Applications, 58 (2009), 1328-1339.

[125] D. Biswas. Blood Flow Models: A Comparative Study. Mittal Publications , 2002.

[126] C. K. Kang, A. C. Eringen. The effect of microstructure on the rheological properties of blood. Bull. Math. Biol., 38 (1976), 135-159.

[127] A. S. Popel, S. A. Regirer. Ob osnovnih uravneniyah hydrodinamiki krovi. Nauchnie trudi instituta mechaniki MGU, 1 (1970), 3-20.

[128] A. S. Popel, S. A. Regirer, P. I. Usick. A Continuum Model of Blood Flow. Biorheology, 11 (1974), 427-437.

[129] A. S. Popel. O hydrodynamike suspensii. Mechanika zjidkosti i gaza, 4 (1969), 24.

[130] A. C. Eringen. Microcontinuum Field Theories II: Fluent media. Springer-Verlag, 2001.

[131] V. A. Levtov, S. A. Regirer, N. Kh. Shadrina. Aggregation and diffusion of Erythrocites. Sovremennie problemi biomekhaniki, 9 (1994), 5-41.

[132] V. L. Kolpashchikov, N. P. Migun, P. P. Prokhorenko. Experimental determination of material micropolar fluid constants. International Journal of Engineering Science, 21 (1983), No. 4, 405-411. 
[133] A. D. J. Kirwan. Boundary conditions for micropolar fluids. International Journal of Engineering Science, 24 (1986), No. 7, 1237-1242.

[134] H. L. Goldsmith, J. C. Marlow. Flow Behavior of Erythrocytes II. Particle Motions in Concentrated Suspensions of Ghost Cells. Journal of Colloid and Interface Science, 71 (1979), No. 2, 383-407.

[135] V. A. Levtov, S. A. Regirer, N. Kh. Shadrina. Rheology of Blood. Medicina, Moscow, 1982.

[136] G. Ahmadi. A continuum theory of blood flow. Scientia Sinica, 24 (1981), No. 10, $1465-$ 1473.

[137] G. Ahmadi. A Continuum Theory for Two Phase Media. Acta Mechanica, 44 (1982), 299317.

[138] J. Jung, D. Gidaspow, I. K. Gamwo. Bubble Computation, Granular Temperatures, and Reynolds Stresses. Chem. Eng. Comm., 193 (2006), 946-975.

[139] E. C. Eckstein, D. G. Bailey, A. H. Shapiro. Self-diffusion of particles in shear flow of a suspension. Journal of Fluid Mechanics, 79 (1977), No. part 1, 191-208.

[140] D. Leighton, A. Acrivos. The shear-induced migration of particles in concentrated suspensions. Journal of Fluid Mechanics, 181 (1987), 415-439.

[141] H. Aref, S. W. Jones. Enhanced separation of diffusing particles by chaotic advection. Phys. Fluids A, 1 (1989), No. 3, 470-474.

[142] C. J. Koh, P. Hookham, L. G. Leal. An experimental investigation of concentrated suspension flows in a rectangular channel. Journal of Fluid Mechanics, 266 (1994), 1-32.

[143] M. K. Lyon, L. G. Leal. An experimental study of the motion of concentrated suspensions in two-dimensional channel flow. Part 1. Monodisperse systems. Journal of Fluid Mechanics, 363 (1998), 25-56.

[144] R. J. Phillips, R. C. Armstrong, R. A. Brown. A constitutive equation for concentrated suspensions that accounts for shear-induced particle migration. Phys. Fluids A, 4 (1992), No. 1, 30-40.

[145] J. E. Butler, R. T. Bonnecaze. Imaging of particle shear migration with electrical impedance tomography. Physics of Fluids, 11 (1999), No. 8, 1982-1994.

[146] M. Hofer, K. Perctold. Computer simulation of concentrated fluid-perticle suspension flows in axisimmetric geometries. Biorheology, 54 (1997), No. 4/5, 261-279.

[147] M. K. Lyon, L. G. Leal. An experimental study of the motion of concentrated suspensions in two-dimensional channel flow. Part 2. Bidisperse systems. Journal of Fluid Mechanics, 363 (1998), 57-77. 
[148] J. F. Morris, F. Boulay. Curvilinear flows of noncolloidal suspensions: The role of normal stresses. Journal of Rheology, 43 (1999), No. 5, 1213-1236.

[149] E. F. Grabowski, Friedman L.I., E. F. Leonard. Effects of Shear Rate on the Diffusion and Adhesion of Blood Platelets to a Foreign Surface. Ind. Eng. Chem. Fundamen., 11 (1972), No. 2, 224-232.

[150] A. B. Strong, G. D. Stubley, G. Chang, D. R. Absolom. Theoretical and experimental analysis of cellular adhesion to polymer surfaces. J. Biomed. Mater. Res., 21 (1987), No. 8, 1039-1055.

[151] G. D. Stubley, A. B. Strong, W. E. Hale, D. R. Absolom. A review of mathematical models for the prediction of blood cell adhesion. PCH PhysicoChem. Hydrodynics, 8 (1987), No. 2, 221-235.

[152] D. M. Wootton, C. P. Markou, S. R. Hanson, D. N. Ku. A mechanistic model of acute platelet accumulation in thrombogenic stenoses. Ann. Biomed. Eng, 29 (2001), No. 4, 321-329.

[153] E. N. Sorensen, G. W. Burgreen, W. R. Wagner, J. F. Antaki. Computational simulation of platelet deposition and activation: II. Results for Poiseuille flow over collagen. Ann. Biomed. Eng, 27 (1999), No. 4, 449-458.

[154] E. N. Sorensen, G. W. Burgreen, W. R. Wagner, J. F. Antaki. Computational simulation of platelet deposition and activation: I. Model development and properties. Ann. Biomed. Eng, 27 (1999), No. 4, 436-448.

[155] T. David, P. G. Walker. Activation and extinction models for platelet adhesion. Biorheology, 39 (2002), 293-298.

[156] M. Anand, K. Rajagopal, K. R. Rajagopal. A Model Incorporating some of the Mechanical and Biochemical Factors Underlying Clot Formation and Dissolution in Flowing Blood. Computational and Mathematical Methods in Medicine, 5 (2003), No. 3\&4, 183-218.

[157] A. L. Fogelson, R. D. Guy. Platelet-wall interactions in continuum models of platelet thrombosis: formulation and numerical solution. Math. Med. Biol., 21 (2004), No. 4, 293-334.

[158] N.-T. Wang, A. L. Fogelson. Computational methods for continuum models of platelet aggregation. Journal of Computational Physics, 151 (1999), 649-675.

[159] A. L. Fogelson. Continuum models of platelet aggregation: formulation and mechanical properties. SIAM J. Appl. Math., 52 (1992), No. 4, 1089-1110.

[160] A. Jordan, T. David, S. Homer-Vanniasinkam, A. Graham, P. Walker. The effects of margination and red cell augmented platelet diffusivity on platelet adhesion in complex flow. Biorheology, 41 (2004), 641-653. 
[161] E. C. Eckstein, D. L. Bilsker, C. M. Waters, J. S. Kippenhan, A. W. Tilles. Transport of platelets in flowing blood. Ann. N. Y. Acad. Sci., 516 (1987), 442-452.

[162] E. C. Eckstein, F. Belgacem. Model of platelet transport in flowing blood with drift and diffusion terms. Biophys. J., 60 (1991), No. 1, 53-69.

[163] C. Yeh, A. C. Calvez, E. C. Eckstein. An estimated shape function for drift in a platelettransport model. Biophys. J., 67 (1994), No. 3, 1252-1259.

[164] A. L. Zydney, C. K. Colton. Augmented solute transport in the shear flow of a concentrated suspension. PCH PhysicoChem. Hydrodynamics, 10 (1988), No. 1, 77-96.

[165] S. N. Antontsev, A. V. Kazhikhov, V. N. Monakov. Boundary Value Problems in the Mechanics of Heterogeneous Fluids, Novosibirsk, Nauka, 1983.

[166] J. Málek, J. Nečas, M. Pokyta, M. Ruzička. Weak and Measure-valued Solutions to Evolutionary DPEs. Chapman and Hall, London, 1996.

[167] G. P. Galdi, R. Rannacher, A. H. Robertson, S. Turek. Hemodynamical Flows Modeling: Analysis and Simulation. Oberwolfach Seminar, Birkhauser, Basel, Boston, Berlin, 2008. 